\title{
Enantioresolution of a Series of Chiral Benzyl Alcohols by HPLC on a Dinitrobenzoylphenylglycine Stationary Phase after Achiral Pre-Column Derivatization*
}

\author{
Svilen P. Simeonov, Anton P. Simeonov, Aleksandar R. Todorov, Vanya B. Kurteva \\ Institute of Organic Chemistry with Centre of Phytochemistry, Bulgarian Academy of Sciences, Sofia, Bulgaria \\ E-mail: \{svilen,art,vkurteva\}@orgchm.bas.bg,aplamenov@gmail.com \\ Received March 24, 2010; revised April 21, 2010; accepted April 23, 2010
}

\begin{abstract}
High performance liquid chromatography method for the separation of a series of chiral benzyl alcohols on $N$-(3,5-dinitrobenzoyl)- $D$-phenylglycine stationary phase (Macherey Nagel, Chiral-2) after pre-column achiral derivatization was developed. Cheap and easy available aromatic acid chlorides were used as derivatization agents. Good to excellent separations of the enantiomers were achieved in all cases in relatively short analytical runs. It was shown that the enantiorecognition depends on the substituents both in the starting alcohol and in the acid chloride. The method presents an efficient alternative to the direct analyses on polysaccharide and cyclodextrine-derived stationary phases.
\end{abstract}

Keywords: HPLC, DNBPG, Enantioseparation, Benzyl Alcohols, Achiral Pre-Column Derivatization, Benzoates, Chlorobenzoates, Naphthoates

\section{Introduction}

Chiral benzyl alcohols are an important class of organic compounds, which exist as structural subunits in many natural and biologically active compounds. They are also widespread products from variety of test transformations used for determination of asymmetric induction caused by different asymmetric catalysts [1-7]. Therefore; the determination of the enantiomeric purity of the products is a crucial step in the preparation of single-enantiomer drugs and chiral catalysts and the development of new and more versatile methods continues to attract attention.

High performance liquid chromatography (HPLC) on chiral stationary phases (CSPs) is among the most general and powerful techniques for separation of optical isomers [8-13]. The efficiency of the separation is strongly dependant on the structure of the stationary phase (SP) used. Of the numerous CSPs available on the market, Brush- type or Pirkle-type columns, containing a low-molecular-weight chiral molecule (chiral selector) covalently bound to the silica gel surface, are the most widely investigated [14-16]. Among them, the phases based on derivatives of $\alpha$-amino acids, inexpensive and readily available enantiomerically pure materials, are the

*Dedicated to the $75^{\text {th }}$ anniversary of Prof. Maria Lyapova most broadly exploited. "Pirkle I-phases" based on dinitrobenzoylphenylglycine (DNBPG) selector, covalently bonded to aminopropyl silica via a spacer, are one of the first and intensively used. The main advantages of these phases are the relatively low cost of the columns and their availability in both enantiomeric forms, which is of great importance for trace analysis where the small peak should be eluted first. However, their application is limited in respect to analyte character. DNBPG has two amide groups, which can undergo dipole-dipole interactions and/or hydrogen bonding with suitable molecules. As these interactions are responsible for the separation, the phases are generally inefficient for direct analysis of some important classes of compounds, such as amines, amino acids, alcohols, amino alcohols, etc.

The enantiomeric distributions of chiral benzyl alcohols were usually analyzed by direct HPLC on polysaccharide [17-25] or cyclodextrine-derived [26-30] stationary phases, while the records on the application of Brush-type CSPs are quite limited. Enantiomers of arylalkylcarbinols have been resolved upon a CSP comprised of DNBPG ionically bonded directly to $\gamma$-aminopropyl silica [31-33]. Sterically congested diarylcarbinols have been resolved by using Pirkle DNBPG ionic or covalent columns and has suggested that the efficient enantiorecognition was achieved due to steric hindrance 
and hydrogen bonding [34]. Phenyl and anthranyl alkanols have been efficiently resolved on SPs with chiral quinidine-carbamate selectors [35-37]. Phases, obtained via immobilization of $(R, R)-3,5$-dinitrobenzoyl-1,2-diphenylethane-1,2-diamine with anchoring groups of varied length and structural type, have been efficiently applied in the enantiorecognition of a series of arylalkylcarbinols [38-42]. Such a CSP, $(S, S)$-Whelk-O 1, has been used both for the direct resolution of diarylmethanols and for their indirect analyzes as acetates and pivalates, the latter being more efficient [43]. Esters, carbonates and carbamates are among the most popular derivatives for indirect resolution of carbinols [44-50]. The diastereoisomers obtained after chiral derivatizations have been analyzed on a variety of phases [51-59], while achirally derivatized carbinols have resolved mainly on polysaccharide-derived CSPs [60-63] and only few articles reported on the application of Pirkle-type phases for the separation of ethers [64], esters $[43,65]$, carbamates [66$68]$. To the best of our knowledge, the enantiorecognition of 3,5-dinitro benzoates [65] is the only record in the literature on the resolution of chiral benzyl alcohols as esters of aromatic acids on DNBPG.

In this paper, we present an effective liquid chromatography method for enantioseparation of benzyl alcohols on one of the cheapest dinitrobenzoylphenylglycine chiral stationary phase (Macherey Nagel, Chiral-2) after achiral pre-column derivatizations as benzoates, chlorobenzoates and naphthoates. The protocol, we believe, is of practical significance as an alternative to the highly efficient direct enantiorecognitions on polysaccharide and cyclodextrinederived phases.

\section{Results and Discussion}

A series of known racemic chiral benzyl alcohols 1-5 was obtained by reduction of the parent ketones with $\mathrm{NaBH}_{4}$ according to a standard procedure. The alcohols were easily converted into the ester derivatives 7-11 by refluxing with an acid chloride in pyridine, as shown on Scheme 1.

These derivatization agents were chosen in an attempt to increase the interactions between the analyte and the $\pi$-acceptor DNBPG stationary phase. Two alternative work-up protocols were applied for the isolation of the

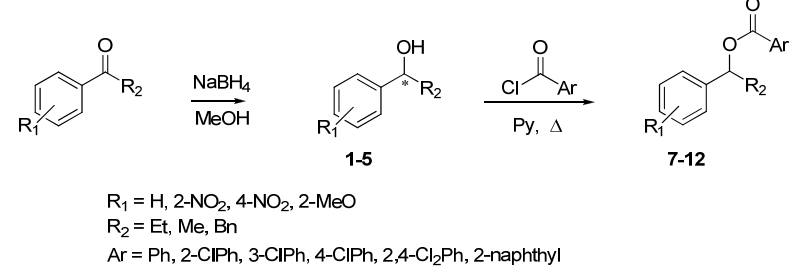

Scheme 1. Preparation of benzyl alcohols 1-5 and derivatives 7-12. products. When ethyl acetate was used for extraction, the esters 7-11 were isolated in high to excellent yields (80-95\%) after purification by HPFC on silica gel. In the second scheme, hexane was used instead of ethyl acetate and the target derivatives were isolated in lower yields $(50-65 \%)$ due to their limited solubility in hexane, but pure enough to be analyzed without chromatography purification, which shortened significantly the general analyzing process. Despite reducing the yield of the esters, the results are explicit as indicated by the same chromatograms obtained after both purification ways. The latter shows that the hexane extraction is the preferable work-up, except for alcohols available in a highly limited scale.

The ester derivatives 7-11 were analyzed by HPLC on Chiral-2 MN column, consisting DNBPG chiral selector, at $25^{\circ} \mathrm{C}$ with mobile phases composed of hexane, $i$-propanol, and trifluoroacetic acid (TFA) in varied proportions. Excellent to very good separations were achieved in all cases (Table 1). The retention factors $k_{1}$ and $k_{2}$ were recalculated towards $T_{0}$, which was determined by using benzene as a standard. All resolution parameters were calculated by the software, adjacent to the apparatus, ChemStation for LC 3D Rev. B.01.01.

As a first series, the esters with phenyl alcohols 1-3, derivatives possessing substituents only at the acid component, were analyzed. The derivatives 7-9 were eluted with hexane/iPr-OH/TFA 100:0.03:0.05 and effective separations were achieved in fast analytical runs, retention times of 5-16 min.

Our expectations were to observe better separation when increasing the $\pi$-character of the molecule; naphthoates vs benzoates, chlorobenzoates vs benzoates, dichlorobenzoates vs monochlorobenzoates. However, benzoates and naphthoates showed commensurable efficiency (Table 1), while a chlorine substituent led to better separation only when ortho-positioned (7b-9b), contrary to 3-chloro and 4-chlorobenzoates (7c-9c and 7d-9d), which were the less effective derivatives. Moreover, the insertion of a second chlorine substituent led to lower resolution, 7e-9e vs $\mathbf{7 b - 9 b}$. The best resolution factors within this series were obtained for 2-chlorobenzoates, 2.86, 2.07 and 1.85 for $7 \mathbf{b}, \mathbf{8 b}$ and $\mathbf{9 b}$, followed by 2,4-dichlorobenzoates, 2.52, 1.82 and 1.48 for 7e, 8e and 9e, respectively. The most effective enantioseparations are illustrated on Figure 1.

As a second series, the esters of alcohols containing nitro-substituent at the aromatic ring, 4 and 5, were obtained and analyzed. To achieve effective combination separation/retention time, more polar mobile phase was used, hexane/iPr-OH/TFA 100:0.1:0.05. As seen on Table 1, the two groups of derivatives, 10a-10f and 11a-11f, follow different separation patterns. Commensurable resolution factors were obtained for the esters with 2-nitrophenyl alcohol 10a-10f. The retention times of 25- 
Table 1. Resolution of the enantiomers of the benzyl alcohol derivatives 7-12.

\begin{tabular}{|c|c|c|c|c|c|c|c|c|}
\hline \multicolumn{3}{|c|}{ Alcohols } & \multicolumn{2}{|c|}{ Derivatives } & \multicolumn{4}{|c|}{ Resolution of the enantiomers ${ }^{\mathrm{a}}$} \\
\hline Compd & $\mathrm{R}_{1}$ & $\mathrm{R}_{2}$ & Compd & $\mathrm{Ar}$ & $k_{1}$ & $k_{2}$ & $\alpha$ & $R_{S}$ \\
\hline \multirow{6}{*}{1} & \multirow{6}{*}{$\mathrm{H}$} & \multirow{6}{*}{$\mathrm{Et}$} & $7 \mathbf{a}$ & $\mathrm{Ph}$ & 2.87 & 3.30 & 1.11 & 2.41 \\
\hline & & & $7 \mathbf{b}$ & 2-ClPh & 4.05 & 4.69 & 1.13 & 2.86 \\
\hline & & & 7c & $3-\mathrm{ClPh}$ & 1.79 & 2.07 & 1.11 & 1.88 \\
\hline & & & $7 d$ & 4- $\mathrm{ClPh}$ & 1.90 & 2.20 & 1.11 & 1.83 \\
\hline & & & $7 e$ & $2,4-\mathrm{Cl}_{2} \mathrm{Ph}$ & 1.78 & 2.10 & 1.11 & 2.52 \\
\hline & & & $7 f$ & 2-naphtyl & 3.82 & 4.39 & 1.12 & 2.48 \\
\hline \multirow{6}{*}{2} & \multirow{6}{*}{$\mathrm{H}$} & \multirow{6}{*}{$\mathrm{Me}$} & $\mathbf{8 a}$ & $\mathrm{Ph}$ & 3.33 & 3.67 & 1.08 & 1.65 \\
\hline & & & $8 \mathbf{b}$ & 2-ClPh & 4.68 & 5.21 & 1.09 & 2.07 \\
\hline & & & $8 c$ & 3-ClPh & 1.22 & 1.38 & 1.07 & 1.12 \\
\hline & & & $8 d$ & 4-ClPh & 0.83 & 0.91 & 1.05 & 1.01 \\
\hline & & & $8 e$ & $2,4-\mathrm{Cl}_{2} \mathrm{Ph}$ & 2.14 & 2.41 & 1.08 & 1.82 \\
\hline & & & $8 f$ & 2-naphtyl & 4.42 & 4.87 & 1.08 & 1.68 \\
\hline \multirow{6}{*}{3} & \multirow{6}{*}{$\mathrm{H}$} & \multirow{6}{*}{$\mathrm{Bn}$} & $9 a$ & $\mathrm{Ph}$ & 3.55 & 3.83 & 1.07 & 1.42 \\
\hline & & & $9 b$ & 2-ClPh & 4.78 & 5.26 & 1.08 & 1.85 \\
\hline & & & $9 c$ & 3-ClPh & 1.55 & 1.67 & 1.05 & 1.08 \\
\hline & & & 9d & 4-ClPh & 2.31 & 2.50 & 1.06 & 1.19 \\
\hline & & & $9 e$ & $2,4-\mathrm{Cl}_{2} \mathrm{Ph}$ & 2.19 & 2.40 & 1.07 & 1.48 \\
\hline & & & $9 f$ & 2-naphtyl & 4.79 & 5.18 & 1.07 & 1.21 \\
\hline \multirow{6}{*}{4} & \multirow{6}{*}{$2-\mathrm{NO}_{2}$} & \multirow{6}{*}{$\mathrm{Me}$} & $10 \mathrm{a}$ & $\mathrm{Ph}$ & 12.67 & 13.73 & 1.08 & 1.73 \\
\hline & & & $10 b$ & 2-ClPh & 8.49 & 9.24 & 1.08 & 1.79 \\
\hline & & & $10 \mathrm{c}$ & 3-ClPh & 8.07 & 8.68 & 1.07 & 1.60 \\
\hline & & & $10 d$ & 4-ClPh & 8.63 & 9.28 & 1.07 & 1.75 \\
\hline & & & $10 \mathrm{e}$ & $2,4-\mathrm{Cl}_{2} \mathrm{Ph}$ & 8.35 & 8.90 & 1.06 & 1.34 \\
\hline & & & $10 \mathrm{f}$ & 2-naphtyl & 18.70 & 20.29 & 1.08 & 1.79 \\
\hline \multirow{6}{*}{5} & \multirow{6}{*}{$4-\mathrm{NO}_{2}$} & \multirow{6}{*}{$\mathrm{Me}$} & 11a & $\mathrm{Ph}$ & 15.33 & 16.26 & 1.06 & 1.34 \\
\hline & & & $11 b$ & 2-ClPh & 8.31 & 8.68 & 1.04 & 0.78 \\
\hline & & & 11c & 3-ClPh & 7.10 & 7.43 & 1.04 & 0.97 \\
\hline & & & 11d & 4-ClPh & 7.13 & 7.49 & 1.04 & 0.97 \\
\hline & & & $11 \mathrm{e}$ & $2,4-\mathrm{Cl}_{2} \mathrm{Ph}$ & 7.93 & 8.25 & 1.04 & 0.78 \\
\hline & & & $11 f$ & 2-naphtyl & 14.34 & 15.25 & 1.06 & 1.32 \\
\hline \multirow{6}{*}{6} & \multirow{6}{*}{ 2-MeO } & \multirow{6}{*}{ Et } & $12 a$ & $\mathrm{Ph}$ & 1.08 & 1.33 & 1.17 & 1.23 \\
\hline & & & $12 b$ & 2-ClPh & 1.70 & 1.97 & 1.13 & 2.11 \\
\hline & & & $12 \mathrm{c}$ & 3-ClPh & 0.76 & 0.93 & 1.15 & 1.65 \\
\hline & & & $12 d$ & 4-ClPh & 0.76 & 0.96 & 1.17 & 1.85 \\
\hline & & & $12 \mathrm{e}$ & $2,4-\mathrm{Cl}_{2} \mathrm{Ph}$ & 0.79 & 0.96 & 1.14 & 1.47 \\
\hline & & & $12 f$ & 2-naphtyl & 1.98 & 2.39 & 1.18 & 3.22 \\
\hline
\end{tabular}

a Flow rate: $1 \mathrm{~mL} / \mathrm{min}$; Detection: $280 \mathrm{~nm} \mathrm{UV}$; Column temperature: $25^{\circ} \mathrm{C}$; Eluent: hexane/iPr-OH/TFA 100:0.03:0.05 for 7-9; hexane/iPr-OH/ TFA 100:0.1:0.05 for 10 and 11; hexane/iPr-OH/TFA 100:0.5:0.05 for 12; $k_{1}$ : retention factor of the first eluted enantiomer; $k_{2}$ : retention factor of the second eluted enantiomer; $\alpha$ : separation factor; $R_{S}$ : resolution factor.

27 min were observed for 10b-10e, while slower elution was detected for 10a and 10f, 36-40 and 53-57 $\mathrm{min}$, respectively. These results show that the monochlorobenzoates 10b-10d are the derivatives of choice within this series, 10b being the preferable example. In the case of the esters with 4-nitrophenyl alcohol $\mathbf{5}$, the simple benzoate 11a and naphthoate 11f showed the best resolution factors, 1.34 and 1.32, respectively (Table 1), while 2chlorobenzoate $\mathbf{1 1 b}$ and 2,4-dichlorobenzoate 11e were the less effective derivatives, 0.78. Thus, 11a and $11 \mathbf{f}$ are the preferred derivatives of $\mathbf{5}$ despite the slower elution process in respect to $\mathbf{1 1 b}-\mathbf{1 1 e}$, $40-46$ vs $21-26 \mathrm{~min}$. The separations of the enantiomers of $\mathbf{1 0 b}$ and $11 \mathrm{a}$ are illustrated on Figure 2.

The method was further extended towards the nonracemic 1-(2-methoxyphenyl)propanol $\mathbf{6}$, obtained by addition of diethylzink to the corresponding aldehyde in the presence of a chiral catalyst by our colleagues [69], who supplied us with a sample. The unknown ester de- rivatives 12a-12f were obtained and purified via the same protocols (Scheme 1) and were afterward analyzed. Relatively polar mobile phase was used, hexane/iPrOH/TFA 100:0.5:0.05, and efficient separations were achieved in very fast elution, 4-8 $\mathrm{min}$. As seen on Table 1, benzoate 12a was the less effective, 1.23, while the best separation was achieved for naphthoate 12f, $R_{S} 3.22$, which is consistent with the initial expectations. Inside chlorinated derivatives, 2-chlorobenzoate 12b showed the best resolution factor, while dichlorosubstituted compound 12e was the less efficient. The chromatograms of the frontier examples $\mathbf{1 2 a}$ and $\mathbf{1 2 f}$ are given on Figure 3.

As seen, the separation is good enough even in the case of 12a to be used for an explicit determination of the enantiomeric excess. The latter is confirmed by the fact that the obtained ee values of the derivatives 12a-12f are in full congruence with the enantiomeric excess of the starting alcohol 6, determined by chiral GC analysis [69]. The 


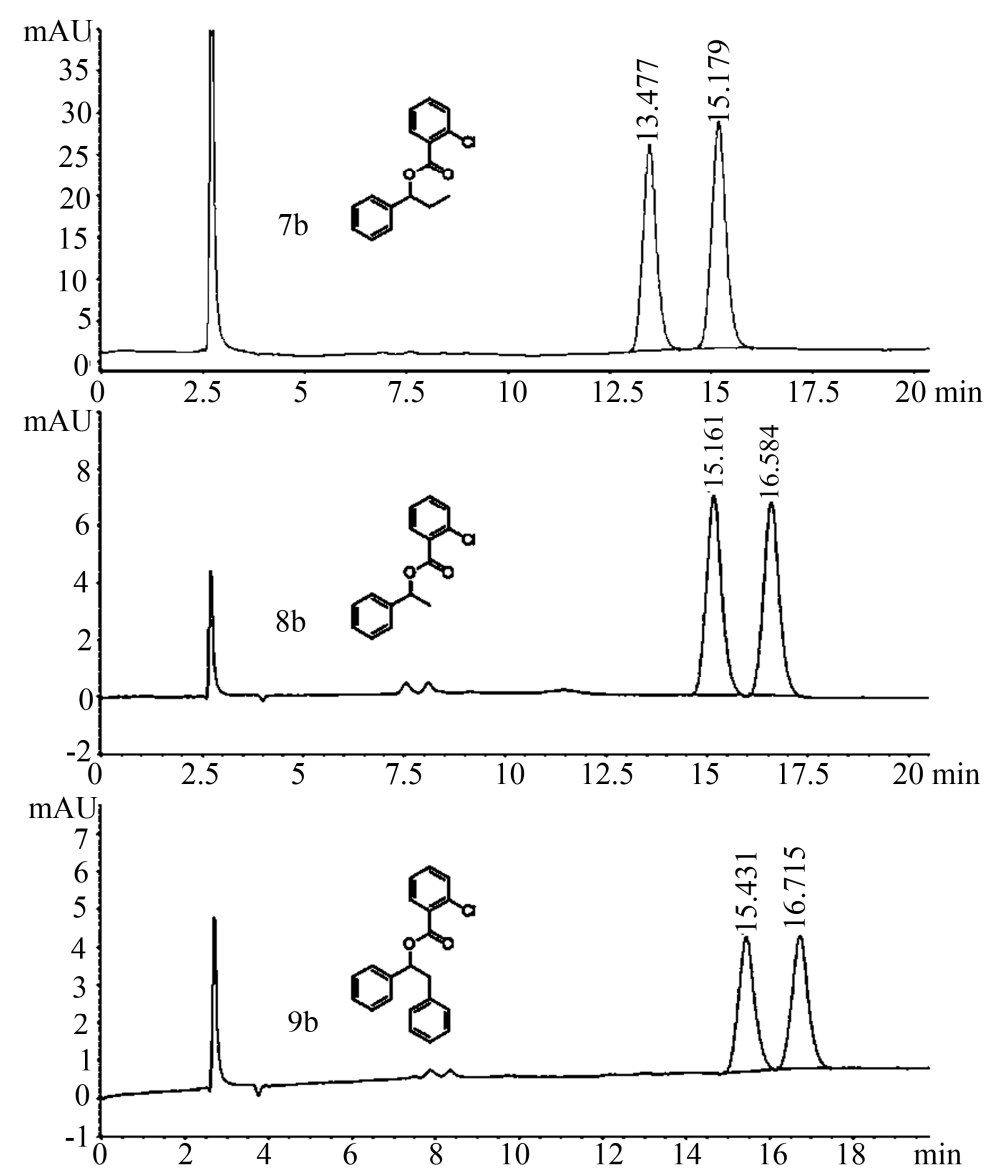

Figure 1. Chromatographic resolution of 1-phenyl-1-alkanol derivatives $7 \mathrm{~b}, 8 \mathrm{~b}$ and $9 \mathrm{~b}$.

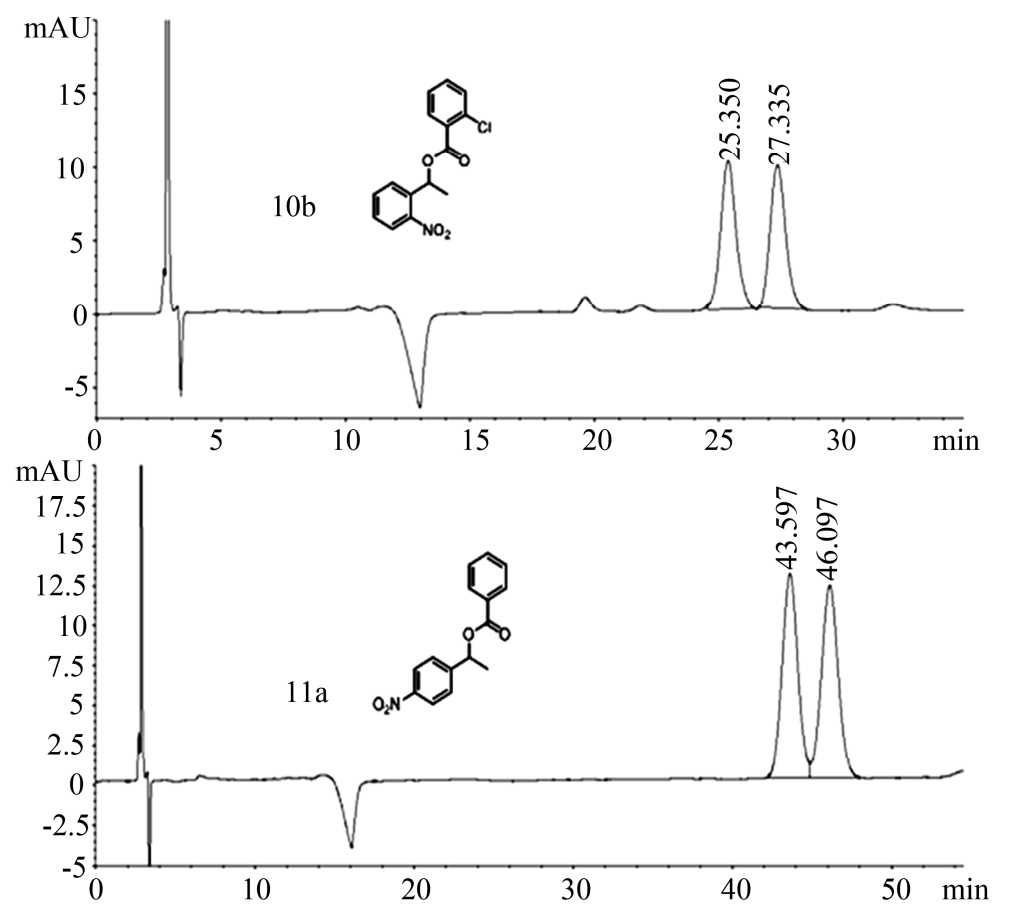

Figure 2. Chromatographic resolution of 1-(nitrophenyl)propanol derivatives $10 \mathrm{~b}$ and $11 \mathrm{a}$ 


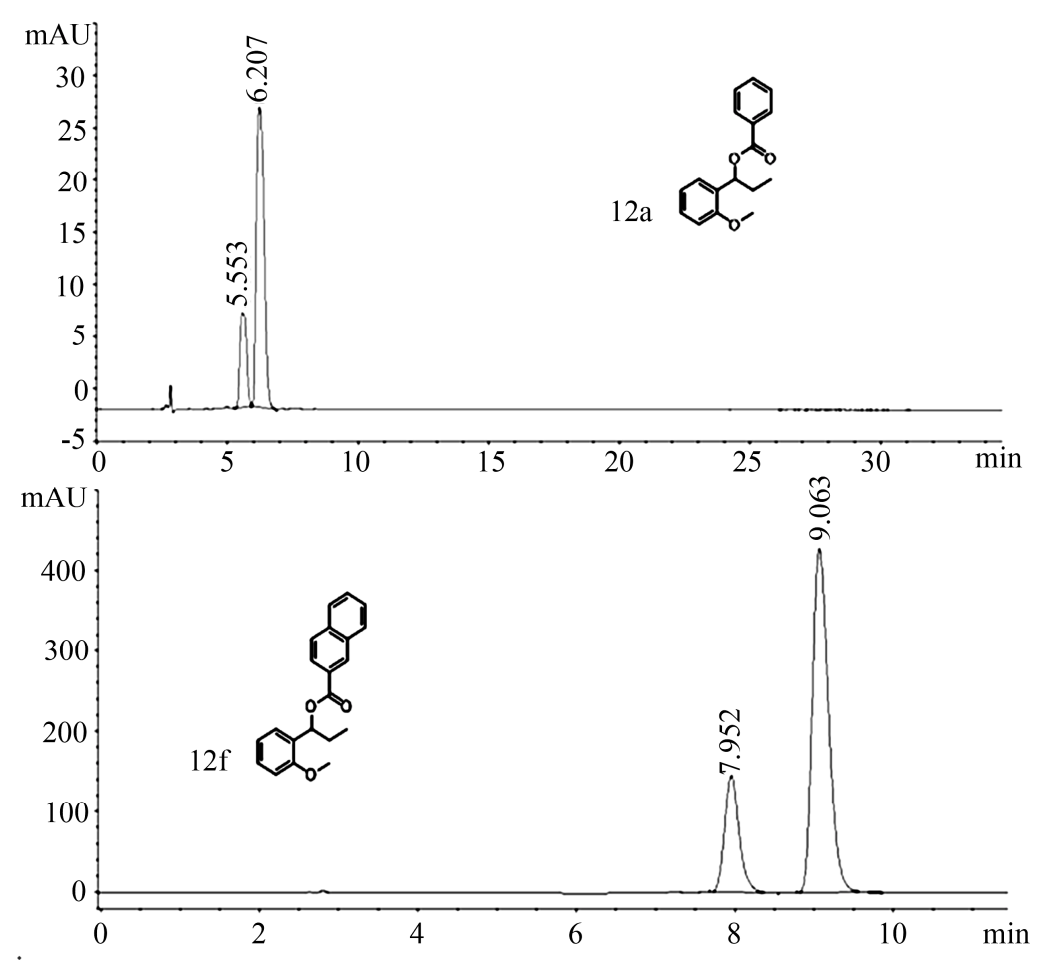

Figure 3. Chromatographic resolution of 1-(2-methoxyphenyl)propanol derivatives 12a and $12 \mathrm{f}$.

appearance of the minor $(R)$-isomer as a first signal presents an additional advantage of the particular analysis protocol especially when high degree of enantioselectivity is achieved. The same pattern is valid for the non-racemic derivatives 7 , where the minor $(R)$-enantiomer elutes first.

\section{Conclusions}

A series of chiral racemic benzyl alcohols and a nonracemic example were analyzed by liquid chromatography on DNBPG stationary phases after achiral pre-column derivatization. Bulk chemistry acid chlorides were used as derivatization agents and the corresponding esters were obtained in high yields after fast and simple synthetic protocol. Good to excellent separations of the enantiomers were achieved in all cases in relatively short analytical runs. The presented method gives possibility to determine the enantiomeric purity or enantioselectivity in the preparation of benzyl alcohols on one of the cheapest and widely exploited stationary phases in a fast, simple, and explicit procedure. Despite being slower than the direct enantiorecognition, we believe, the protocol should be useful to the synthetic community as an alternative way, especially when other chiral columns are not available in the laboratory. Additionally, the obtained broad library of chiral benzyl alcohol esters offers possibility to select a convenient derivative according to the available reagents.

\section{Experimental}

General: All reagents were purchased from Aldrich, Merck and Fluka and were used without any further purification. Fluka silica gel/TLC-cards 60778 with fluorescent indicator $254 \mathrm{~nm}$ were used for TLC chromatography and Rf-values determination. The high performance flash chromatography (HPFC) purifications were carried out on a Biotage HorizonTM system (Charlottesville, Virginia, USA) on silica gel. The melting points were determined in capillary tubes on SRS MPA100 OptiMelt (Sunnyvale, CA, USA) automated melting point system. The NMR spectra were recorded on a Bruker Avance DRX 250 and Bruker Avance II+ 600 (where indicated) spectrometers (Rheinstetten, Germany) in deuterochloroform; the chemical shifts were quoted in ppm in $\delta$-values against tetramethylsilane (TMS) as an internal standard and the coupling constants were calculated in $\mathrm{Hz}$.

The high performance liquid chromatography (HPLC) enantioseparations were performed on an Agilent 1100 System fitted with diode array detector and manual injector with a $20 \mu \mathrm{L}$ injection loop. A stainless-steel Nucleosil Chiral-2 column (Macherey-Nagel GmbH \&Co. KG, Düren, Germany) was used; $250 \times 4 \mathrm{~mm}$, particle size $5 \mu \mathrm{m}$, pore size $100 \AA$, chiral selector $N$-(3,5-dinitrobenzoyl)- $D$-phenylglycine. The analyses were performed at $25^{\circ} \mathrm{C}$ with a flow rate of $1.0 \mathrm{~mL} / \mathrm{min}$. The HPLC grade solvents were purchased from 
Sigma-Aldrich and Labscan.

Synthesis of chiral racemic benzyl alcohols 1-5: To a solution of a ketone $(20 \mathrm{mmol})$ in $\mathrm{MeOH}(20 \mathrm{~mL}) \mathrm{NaBH}_{4}$ (30 mmol) was added portionwise and the mixture was stirred at room temperature for 0.5-1 h. The solvent was removed in vacuo and the products were partitioned between water and $\mathrm{CH}_{2} \mathrm{Cl}_{2}$. The organic layer was dried over $\mathrm{Na}_{2} \mathrm{SO}_{4}$, evaporated to dryness, and purified by HPFC on silica gel.

1-Phenyl-1-propanol 1 [70]: 92\% yield; $\mathrm{R}_{\mathrm{f}} 0.48$ (hexane:EtOAc 80:20); ${ }^{1} \mathrm{H}$ NMR 0.88 (t, 3H, J 7.4, $\left.\mathrm{CH}_{3}\right), 1.75$ (m, 2H, $\left.\mathrm{CH}_{2}\right), 2.24$ (bd, 1H, J 1.7, OH), 4.53 (td, 1H, J 1.7, 7.3, CH), $7.32(\mathrm{~m}, 5 \mathrm{H}, \mathrm{CH}-\mathrm{Ph})$.

1-Phenylethanol 2 [71]: 93\% yield; $\mathrm{R}_{\mathrm{f}} 0.38$ (hexane: EtOAc 80:20); ${ }^{1} \mathrm{H}$ NMR 1.47 (d, 3H, J 6.5, $\left.\mathrm{CH}_{3}\right), 2.12$ (bs, 1H, OH), 4.85 (q, 1H, J 6.5, 12.9, CH), 7.33 (m, 5H, $\mathrm{CH}$-Ph).

1,2-Diphenylethanol 3 [72]: 59\% yield; $\mathrm{R}_{\mathrm{f}} 0.48$ (hexane: EtOAc 80:20); ${ }^{1} \mathrm{H}$ NMR 2.02 (bs, 1H, OH), 2.98 (m, 2H, $\left.\mathrm{CH}_{2}\right), 4.86(\mathrm{dd}, 1 \mathrm{H}, \mathrm{J} 5.5,7.8, \mathrm{CH}), 7.24(\mathrm{~m}, 10 \mathrm{H}$, $\mathrm{CH}-\mathrm{Ph})$.

1-(2-Nitrophenyl)ethanol 4 [73]: $88 \%$ yield; $\mathrm{R}_{\mathrm{f}} 0.35$ $\left(\mathrm{CH}_{2} \mathrm{Cl}_{2}\right) ;{ }^{1} \mathrm{H}$ NMR 1.52 (d, 3H, J 6.4, $\left.\mathrm{CH}_{3}\right), 2.99$ (s, 1H, $\mathrm{OH}$ ), 5.37 (q, 1H, J 6.2, 12.5, CH), 7.39 (ddd, 1H, J 1.5, 7.4, 8.1, CH-Ar), 7.62 (ddd, 1H, J 1.3, 7.4, 7.9, CH-Ar), 7.80 (dd, 1H, J 1.5, 7.9, CH-Ar), 7.85 (dd, 1H, J 1.3, 8.1, $\mathrm{CH}$-Ar).

1-(4-Nitrophenyl)ethanol 5 [74]: 97\% yield; $\mathrm{R}_{\mathrm{f}} 0.40$ $\left(\mathrm{CH}_{2} \mathrm{Cl}_{2}\right) ;{ }^{1} \mathrm{H}$ NMR 1.52 (d, 3H, J 6.5, $\left.\mathrm{CH}_{3}\right), 2.26$ (bd, $1 \mathrm{H}, \mathrm{J} 3.4, \mathrm{OH}), 5.02(\mathrm{qd}, 1 \mathrm{H}, \mathrm{J} 3.4,6.5,13.0, \mathrm{CH}), 7.54$ (dt, 2H, J 2.4, 8.8, CH-Ar), 8.18 (dt, 2H, J 2.4, 8.8, $\mathrm{CH}$-Ar).

Synthesis of the derivatives 7-12: To a solution of a benzyl alcohol 1-6 (1 mmol) in pyridine $(2 \mathrm{~mL})$ an acid chloride $(1.1 \mathrm{mmol})$ was added and the mixture was refluxed for $30 \mathrm{~min}$. Sat. aq. $\mathrm{K}_{2} \mathrm{CO}_{3}$ was added and the mixture was stirred for $15 \mathrm{~min}$ at room temperature. Work-up:

Method 1: The products were partitioned between water and EtOAc. The organic layer was dried over $\mathrm{Na}_{2} \mathrm{SO}_{4}$, evaporated to dryness, and purified by HPFC on silica gel.

Method 2: The products were partitioned between water and hexane. The organic layer was dried over $\mathrm{Na}_{2} \mathrm{SO}_{4}$ and evaporated to dryness.

1-Phenylpropyl benzoate 7a [75]: 91\% yield; $\mathrm{R}_{\mathrm{f}} 0.56$ $\left(\mathrm{CH}_{2} \mathrm{Cl}_{2}\right) ;{ }^{1} \mathrm{H}$ NMR 0.94 (t, 3H, J 7.4, $\left.\mathrm{CH}_{3}\right), 1.99(\mathrm{~m}, 2 \mathrm{H}$, $\left.\mathrm{CH}_{2}\right), 5.94$ (t, 1H, J 6.8, CH-O), 7.35 (m, 8H, CH-Ar), 8.08 (m, 2H, CH-Ar); ${ }^{13} \mathrm{C}$ NMR $9.8\left(\mathrm{CH}_{3}\right), 29.4\left(\mathrm{CH}_{2}\right)$, 77.7 (CH-O), 126.3 (2 CH-Ar), 127.7 (CH-Ar), 128.2 (2 CH-Ar), 128.3 (2 CH-Ar), 129.4 (2 CH-Ar), 130.4 ( $\left.C_{\text {quat }}\right)$, 132.7 (CH-Ar), $140.5\left(C_{\text {quat }}\right), 165.6(C=\mathrm{O})$; HPLC: eluent hexane/iPr-OH/TFA 100:0.03:0.05, retention times $t_{R}-1$ 10.33 and $t_{R}-211.47 \mathrm{~min}$.

1-Phenylpropyl 2-chlorobenzoate 7b: $82 \%$ yield; $\mathrm{R}_{\mathrm{f}}$ $0.72\left(\mathrm{CH}_{2} \mathrm{Cl}_{2}\right) ;{ }^{1} \mathrm{H}$ NMR 0.94 (t, 3H, J 7.4, $\left.\mathrm{CH}_{3}\right), 2.00$ (m, $\left.2 \mathrm{H}, \mathrm{CH}_{2}\right), 5.94(\mathrm{t}, 1 \mathrm{H}, \mathrm{J}$ 6.8, $\mathrm{CH}-\mathrm{O}), 7.26(\mathrm{~m}, 8 \mathrm{H}$, $\mathrm{CH}$-Ar), 7.80 (dd, 1H, J 1.9, 7.6, CH-Ar); ${ }^{13} \mathrm{C}$ NMR 9.8 $\left(\mathrm{CH}_{3}\right), 29.2\left(\mathrm{CH}_{2}\right), 78.6(\mathrm{CH}-\mathrm{O}), 126.3(\mathrm{CH}-\mathrm{Ar}), 126.5$ (2 CH-Ar), 127.7 (CH-Ar), 128.2 (2 CH-Ar), 130.2 $\left(C_{\text {quat }}\right), 130.8$ (CH-Ar), 131.2 (CH-Ar), 132.2 (CH-Ar), $133.4\left(C_{\text {quat }}\right), 139.9\left(C_{\text {quat }}\right), 164.7(C=\mathrm{O})$; HPLC: eluent hexane/iPr-OH/TFA 100:0.03:0.05, retention times $t_{R}-1$ 13.48 and $t_{R}-215.18 \mathrm{~min}$.

1-Phenylpropyl 3-chlorobenzoate 7c: $81 \%$ yield; $\mathrm{R}_{\mathrm{f}}$ 0.65 (hexane: $\mathrm{CH}_{2} \mathrm{Cl}_{2}$ 60:40); m. p. $63-64{ }^{\circ} \mathrm{C} ;{ }^{1} \mathrm{H} \mathrm{NMR}$ 0.96 (t, 3H J 7.4, $\left.\mathrm{CH}_{3}\right), 2.02\left(2 \mathrm{H}, \mathrm{m}, \mathrm{CH}_{2}\right), 5.91(\mathrm{t}, 1 \mathrm{H}, \mathrm{J}$ 6.8, CH-O), 7.35 (m, 6H, CH-Ar), 7.52 (ddd, 1H, J 1.2, 2.0, 8.0, CH-Ar), 7.96 (dt, 1H, J 1.3, 7.7, CH-Ar), 8.04 (t, 1H, J 1.8, CH-Ar); ${ }^{13} \mathrm{C}$ NMR $9.9\left(\mathrm{CH}_{3}\right), 29.4\left(\mathrm{CH}_{2}\right), 78.5$ (CH-O), 126.5 (2 CH-Ar), 127.8 (CH-Ar), 128.0 (CH-Ar), 128.5 (2 CH-Ar), 129.6 (CH-Ar), 129.7 $(C H-A r), 132.3\left(C_{\text {quat }}\right), 132.9$ (CH-Ar), $134.5\left(C_{\text {quat }}\right)$, $140.2\left(C_{\text {quat }}\right), 164.7(C=\mathrm{O})$; HPLC: eluent hexane/iPr-OH/ TFA 100:0.03:0.05, retention times $t_{R}-17.45$ and $t_{R}-2$ $8.20 \mathrm{~min}$.

1-Phenylpropyl 4-chlorobenzoate 7d: $60 \%$ yield; $\mathrm{R}_{\mathrm{f}}$ 0.53 (hexane:EtOAc 90:10); ${ }^{1} \mathrm{H}$ NMR 0.95 (t, 3H, J 7.4, $\left.\mathrm{CH}_{3}\right), 2.01\left(\mathrm{~m}, 2 \mathrm{H}, \mathrm{CH}_{2}\right), 5.91$ (t, 1H, J 6.8, CH-O), 7.31 (m, 7H, CH-Ar), 8.01 (dt, 2H, J 2.3, 9.0, CH-Ar); ${ }^{13} \mathrm{C}$ NMR $9.9\left(\mathrm{CH}_{3}\right), 29.4\left(\mathrm{CH}_{2}\right), 78.2(\mathrm{CH}-\mathrm{O}), 126.4(2$ CH-Ar), 127.9 (CH-Ar), 128.4 (2 CH-Ar), 128.6 (2 CH-Ar), $129.0\left(C_{\text {quat }}\right), 131.0$ (2 CH-Ar), $139.3\left(C_{\text {quat }}\right)$, $140.3\left(C_{\text {quat }}\right), 165.0(C=\mathrm{O})$; HPLC: eluent hexane/ iPr-OH/TFA 100:0.03:0.05, retention times $t_{R}-17.73$ and $\mathrm{t}_{\mathrm{R}}-28.54 \mathrm{~min}$.

1-Phenylpropyl 2,4-dichlorobenzoate 7e: $96 \%$ yield; $\mathrm{R}_{\mathrm{f}} 0.72\left(\mathrm{CH}_{2} \mathrm{Cl}_{2}\right) ;{ }^{1} \mathrm{H}$ NMR 0.94 (t, 3H, J 7.4, $\left.\mathrm{CH}_{3}\right), 2.00$ $\left(\mathrm{m}, 2 \mathrm{H}, \mathrm{CH}_{2}\right), 5.92$ (t, 1H, J 6.8, CH-O), 7.23 (dd, 1H, J 2.0, 8.4, CH-Ar), 7.33 (m, 5H, CH-Ar), 7.41 (d, 1H, J 2.0, CH-Ar), 7.79 (d, 1H, J 8.4, CH-Ar); ${ }^{13} \mathrm{C}$ NMR 9.8 $\left(\mathrm{CH}_{3}\right), 29.2\left(\mathrm{CH}_{2}\right), 79.0(\mathrm{CH}-\mathrm{O}), 126.5$ (2 $\left.\mathrm{CH}-\mathrm{Ar}\right)$, 126.8 (CH-Ar), 127.9 (CH-Ar), 128.3 (2 CH-Ar), 128.5 $\left(C_{\text {quat }}\right), 130.8(\mathrm{CH}-\mathrm{Ar}), 132.4(\mathrm{CH}-\mathrm{Ar}), 134.7\left(C_{\text {quat }}\right)$, $138.0\left(C_{\text {quat }}\right), 139.8\left(C_{\text {quat }}\right), 163.9(C=\mathrm{O})$; HPLC: eluent hexane/iPr-OH/TFA 100:0.03:0.05, retention times $t_{R}-1$ 7.43 and $t_{R}-28.27$ min.

1-Phenylpropyl 2-naphthoate 7f [76]: 79\% yield; $\mathrm{R}_{\mathrm{f}}$ $0.80\left(\mathrm{CH}_{2} \mathrm{Cl}_{2}\right) ;{ }^{1} \mathrm{H} \mathrm{NMR} 0.97$ (t, 3H J 7.4, $\left.\mathrm{CH}_{3}\right), 2.04$ $\left(\mathrm{m}, 2 \mathrm{H}, \mathrm{CH}_{2}\right), 6.01(\mathrm{t}, 1 \mathrm{H}, \mathrm{J}$ 6.8, CH-O), $7.30(\mathrm{~m}, 3 \mathrm{H}$, $\mathrm{CH}$-Ar), 7.44 (m, 4H, CH-Ar), 7.77 (dd, 2H, J 7.0, 8.6, $\mathrm{CH}$-Ar), 7.87 (dd, 1H, J 2.2, 6.9, CH-Ar), 8.10 (dd, 1H, J 1.7, 8.6, CH-Ar), 8.63 (s, $1 \mathrm{H}, \mathrm{CH}-\mathrm{Ar}) ;{ }^{13} \mathrm{C}$ NMR 9.8 $\left(\mathrm{CH}_{3}\right), 29.4\left(\mathrm{CH}_{2}\right), 77.8(\mathrm{CH}-\mathrm{O}), 125.1(\mathrm{CH}-\mathrm{Ar}), 126.3$ (2 CH-Ar), 126.4 (CH-Ar), 127.5 (CH-Ar), $127.6\left(C_{\text {quat }}\right)$, 127.7 (CH-Ar), 127.9 (CH-Ar), 128.0 (CH-Ar), 128.3 (2 CH-Ar), 129.1 (CH-Ar), 130.8 (CH-Ar), 132.3 ( $\left.C_{\text {quat }}\right)$, $135.3\left(C_{\text {quat }}\right), 140.5\left(C_{\text {quat }}\right), 165.8(C=\mathrm{O})$; HPLC: eluent hexane/iPr-OH/TFA 100:0.03:0.05, retention times $t_{R}-1$ 12.87 and $t_{R}-214.39 \mathrm{~min}$.

1-Phenylethyl benzoate 8a [77]: $51 \%$ yield; $\mathrm{R}_{\mathrm{f}} 0.40$ (hexane: $\mathrm{CH}_{2} \mathrm{Cl}_{2}$ 60:40); ${ }^{1} \mathrm{H}$ NMR 1.66 (d, 3H, J 6.6, 
$\left.\mathrm{CH}_{3}\right), 6.14$ (q, 1H, J 6.6, 13.2, $\left.\mathrm{CH}-\mathrm{O}\right), 7.38$ (m, 8H, $\mathrm{CH}$-Ar), 8.08 (m, 2H, CH-Ar); ${ }^{13} \mathrm{C} \mathrm{NMR} 22.3\left(\mathrm{CH}_{3}\right)$, 72.8 (CH-O), 126.0 (2 CH-Ar), 127.8 (CH-Ar), 128.3 (2 CH-Ar), 128.5 (2 CH-Ar), 129.6 (2 CH-Ar), 130.5 $\left(C_{\text {quat }}\right), 132.8$ (CH-Ar), $141.7\left(C_{\text {quat }}\right), 165.7(C=\mathrm{O})$; HPLC: eluent hexane/iPr-OH/TFA 100:0.03:0.05, retention times $t_{R}-111.56$ and $t_{R}-212.47 \mathrm{~min}$.

1-Phenylethyl 2-chlorobenzoate 8b: $45 \%$ yield; $\mathrm{R}_{\mathrm{f}}$ 0.59 (hexane: $\left.\mathrm{CH}_{2} \mathrm{Cl}_{2} 60: 40\right) ;{ }^{1} \mathrm{H}$ NMR 1.68 (d, 3H, J 6.6, $\left.\mathrm{CH}_{3}\right), 6.14$ (q, 1H, J 6.6, 13.2, $\left.\mathrm{CH}-\mathrm{O}\right), 7.35$ (m, 8H, $\mathrm{CH}$-Ar), 7.82 (ddd, $1 \mathrm{H}, \mathrm{J}$ 0.5, 1.7, 7.7, CH-Ar); ${ }^{13} \mathrm{C}$ NMR $22.2\left(\mathrm{CH}_{3}\right), 73.8$ (CH-O), 126.2 (2 CH-Ar), 126.5 (CH-Ar), 128.0 (CH-Ar), 128.5 (2 CH-Ar), 130.4 ( $\left.C_{\text {quat }}\right)$, 131.0 (CH-Ar), 131.3 (CH-Ar), 132.4 (CH-Ar), 133.6 $\left(C_{\text {quat }}\right), 141.2\left(C_{\text {quat }}\right), 164.9(C=\mathrm{O})$; HPLC: eluent hexane/ iPr-OH/TFA 100:0.03:0.05, retention times $t_{R}-115.16$ and $\mathrm{t}_{\mathrm{R}}-216.58 \mathrm{~min}$.

1-Phenylethyl 3-chlorobenzoate 8c [78]: 48\% yield; $\mathrm{R}_{\mathrm{f}}$ 0.64 (hexane: $\mathrm{CH}_{2} \mathrm{Cl}_{2} 60: 40$ ); m. p. $134-136{ }^{\circ} \mathrm{C} ;{ }^{1} \mathrm{H}$ NMR 1.69 (d, 3H, J 6.6, $\left.\mathrm{CH}_{3}\right), 6.14$ (dd, $1 \mathrm{H}, \mathrm{J}$ 6.6, 13.2, CH-O), 7.41 (m, 6H, CH-Ar), 7.53 (ddd, 1H, J 1.2, 2.1, 8.0, CH-Ar), 7.96 (dt, 1H, J 1.4, 7.8, CH-Ar), 8.05 (t, 1H, J 2.0, CH-Ar); ${ }^{13} \mathrm{C}$ NMR $22.2\left(\mathrm{CH}_{3}\right), 73.5(\mathrm{CH}-\mathrm{O}), 126.1$ (2 CH-Ar), 127.8 (CH-Ar) 128.0 (CH-Ar) 128.6 (2 CH-Ar), 129.6 (CH-Ar), 132.3 (CH-Ar), 132.9 (CH-Ar), $133.7\left(C_{\text {quat }}\right), 134.5\left(C_{\text {quat }}\right), 141.4\left(C_{\text {quat }}\right), 164.6(C=\mathrm{O})$; HPLC: eluent hexane/iPr-OH/TFA 100:0.03:0.05, retention times $t_{R}-15.94$ and $t_{R}-26.35$ min.

1-Phenylethyl 4-chlorobenzoate 8d: 99\% yield; $\mathrm{R}_{\mathrm{f}}$ 0.72 (hexane: $\mathrm{CH}_{2} \mathrm{Cl}_{2}$ 40:60); ${ }^{1} \mathrm{H}$ NMR 1.69 (d, 3H, J 6.6, $\left.\mathrm{CH}_{3}\right), 6.14$ (dd, $1 \mathrm{H}, \mathrm{J}$ 6.6, 13.2, $\left.\mathrm{CH}-\mathrm{O}\right) 7.37$ (m, 7H, $\mathrm{CH}$-Ar), 8.02 (ddd, 2H, J 2.0, 2.3, 8.4, CH-Ar); ${ }^{13} \mathrm{C}$ NMR $22.3\left(\mathrm{CH}_{3}\right), 73.2$ (CH-O), 126.0 (2 $\left.\mathrm{CH}-\mathrm{Ar}\right), 128.0$ (CH-Ar), 128.5 (2 CH-Ar), 128.6 (2 CH-Ar), 129.0 $\left(C_{\text {quat }}\right), 131.0$ (2 CH-Ar), $139.3\left(C_{\text {quat }}\right), 141.5\left(C_{\text {quat }}\right)$, $164.9 \quad(C=\mathrm{O})$; HPLC: eluent hexane/iPr-OH/TFA 100:0.03:0.05, retention times $t_{R}-14.88$ and $t_{R}-2 \quad 5.10$ $\min$.

1-Phenylethyl 2,4-dichlorobenzoate 8e: $86 \%$ yield; $\mathrm{R}_{\mathrm{f}}$ 0.70 (hexane: $\left.\mathrm{CH}_{2} \mathrm{Cl}_{2} 60: 40\right) ;{ }^{1} \mathrm{H}$ NMR 1.67 (d, 3H, J 6.6, $\mathrm{CH}_{3}$ ), 6.12 (q, 1H, J 6.6, 13.2, $\left.\mathrm{CH}-\mathrm{O}\right), 7.34$ (m, 7H, $\mathrm{CH}$-Ar), 7.79 (d, 1H, J 8.4, CH-Ar); ${ }^{13} \mathrm{C}$ NMR 22.2 $\left(\mathrm{CH}_{3}\right), 74.1$ (CH-O), 126.2 (2 $\left.\mathrm{CH}-\mathrm{Ar}\right), 126.9$ (CH-Ar), 128.1 (CH-Ar), 128.5 (2 CH-Ar), $128.6\left(C_{\text {quat }}\right), 130.9$ $(C H-A r), 132.5$ (CH-Ar), $134.9\left(C_{\text {quat }}\right), 138.2\left(C_{\text {quat }}\right)$, $141.0\left(C_{\text {quat }}\right), 164.0(C=\mathrm{O})$; HPLC: eluent hexane/iPr-OH/ TFA 100:0.03:0.05, retention times $t_{R}-18.39$ and $t_{R}-2$ $9.10 \mathrm{~min}$.

1-Phenylethyl 2-naphthoate 8f [79]: 50\% yield; $\mathrm{R}_{\mathrm{f}}$ 0.63 (hexane: $\mathrm{CH}_{2} \mathrm{Cl}_{2}$ 40:60); ${ }^{1} \mathrm{H}$ NMR 1.71 (d, 3H, J 6.6, $\left.\mathrm{CH}_{3}\right), 6.20$ (q, 1H, J 6.6, 13.2, $\left.\mathrm{CH}-\mathrm{O}\right), 7.32$ (m, 3H, $\mathrm{CH}$-Ar), 7.50 (m, 4H, CH-Ar), 7.82 (m, 2H, CH-Ar), 7.91 (dd, 1H, J 1.7, 7.3, CH-Ar), 8.09 (dd, 1H, J 1.7, 8.6, $\mathrm{CH}$-Ar), 8.63 (s, 1H, CH-Ar); ${ }^{13} \mathrm{C}$ NMR $22.3\left(\mathrm{CH}_{3}\right), 73.0$ (CH-O), 125.2 (CH-Ar), 126.0 (2 CH-Ar), 126.5
(CH-Ar), 127.6 (CH-Ar), 127.7 ( $\left.C_{\text {quat }}\right), 127.8$ (CH-Ar), 128.0 (CH-Ar), 128.1 (CH-Ar), 128.5 (2 CH-Ar) 129.3 (CH-Ar), 131.0 (CH-Ar), $132.4\left(C_{\text {quat }}\right), 135.4\left(C_{\text {quat }}\right)$, $141.8\left(C_{\text {quat }}\right), 165.9(C=\mathrm{O})$; HPLC: eluent hexane/iPr-OH/ TFA 100:0.03:0.05, retention times $t_{R}-114.48$ and $t_{R}-2$ $15.68 \mathrm{~min}$.

1,2-Diphenylethyl benzoate 9a [80]: 73\% yield; $\mathrm{R}_{\mathrm{f}} 0.43$ (hexane:EtOAc 90:10); m. p. 67-68 ${ }^{\circ} \mathrm{C}$ (lit. [80] 70 ${ }^{\circ} \mathrm{C}$ ); ${ }^{1} \mathrm{H}$ NMR 3.19 (A part of ABX, 1H, J $\mathrm{J}_{\mathrm{AX}} 6.0, \mathrm{~J}_{\mathrm{AB}} 13.8,1 / 2$ $\mathrm{CH}_{2}$ ), 3.35 (B part of $\mathrm{ABX}, 1 \mathrm{H}, \mathrm{J}_{\mathrm{BX}} 7.8, \mathrm{~J}_{\mathrm{AB}} 13.8,1 / 2$ $\left.\mathrm{CH}_{2}\right), 6.18(\mathrm{dd}, 1 \mathrm{H}, \mathrm{J} 6.0,7.6, \mathrm{CH}-\mathrm{O}), 7.18$ (m, 5H, $\mathrm{CH}$-Ar), 7.30 (m, 5H, CH-Ar), 7.41 (m, 2H, CH-Ar), 7.53 (m, 1H, CH-Ar), 8.04 (m, 2H, CH-Ar); ${ }^{13} \mathrm{C}$ NMR $43.2\left(\mathrm{CH}_{2}\right), 77.2(\mathrm{CH}-\mathrm{O}), 126.5(2 \mathrm{CH}-\mathrm{Ar}), 126.6$ (CH-Ar), 127.9 (CH-Ar), 128.2 (2 CH-Ar), 128.3 (2 CH-Ar), 128.4 (2 CH-Ar), 129.6 (4 CH-Ar), 130.4 ( $\left.C_{\text {quat }}\right)$, 132.9 (CH-Ar), $136.9\left(C_{\text {quat }}\right), 140.1\left(C_{\text {quat }}\right), 165.6(C=\mathrm{O})$; HPLC: eluent hexane/iPr-OH/TFA 100:0.03:0.05, retention times $t_{R}-112.14$ and $t_{R}-212.97 \mathrm{~min}$.

1,2-Diphenylethyl 2-chlorobenzoate 9b: 65\% yield; $\mathrm{R}_{\mathrm{f}}$ 0.63 (hexane:EtOAc 90:10); ${ }^{1} \mathrm{H}$ NMR 3.19 (A part of $\mathrm{ABX}, 1 \mathrm{H}, \mathrm{J}_{\mathrm{AX}} 6.4, \mathrm{~J}_{\mathrm{AB}} 13.7,1 / 2 \mathrm{CH}_{2}$ ), 3.36 (B part of $\left.\mathrm{ABX}, 1 \mathrm{H}, \mathrm{J}_{\mathrm{BX}} 7.6, \mathrm{~J}_{\mathrm{AB}} 13.7,1 / 2 \mathrm{CH}_{2}\right), 6.20(\mathrm{dd}, 1 \mathrm{H}, \mathrm{J} 6.4$, 7.6, CH-O), 7.28 (m, 13H, CH-Ar), 7.73 (ddd, 1H, J 0.6, 1.5, 8.0, CH-Ar); ${ }^{13} \mathrm{C}$ NMR $43.0\left(\mathrm{CH}_{2}\right), 78.1$ ( $\left.\mathrm{CH}-\mathrm{O}\right)$, 126.5 (CH-Ar), 126.6 (CH-Ar), 126.7 (2 CH-Ar), 128.1 (CH-Ar), 128.3 (2 CH-Ar), 128.4 (2 CH-Ar), 129.6 (2 CH-Ar), 130.2 ( $\left.C_{\text {quat }}\right), 131.0$ (CH-Ar), 131.4 (CH-Ar), 132.4 (CH-Ar), $133.8\left(C_{\text {quat }}\right), 136.7\left(C_{\text {quat }}\right), 139.6\left(C_{\text {quat }}\right)$, $164.7 \quad(C=\mathrm{O})$; HPLC: eluent hexane/iPr-OH/TFA 100:0.03:0.05, retention times $t_{R}-115.43$ and $t_{R}-216.72$ $\min$.

1,2-Diphenylethyl 3-chlorobenzoate 9c: $56 \%$ yield; $\mathrm{R}_{\mathrm{f}}$ 0.40 (hexane:EtOAc 90:10); m. p. $73-74^{\circ} \mathrm{C}$; ${ }^{1} \mathrm{H}$ NMR 3.19 (A part of $\mathrm{ABX}, 1 \mathrm{H}, \mathrm{J}_{\mathrm{AX}} 6.1, \mathrm{~J}_{\mathrm{AB}} 13.8,1 / 2 \mathrm{CH}_{2}$ ), 3.35 (B part of $\left.\mathrm{ABX}, 1 \mathrm{H}, \mathrm{J}_{\mathrm{BX}} 7.7, \mathrm{~J}_{\mathrm{AB}} 13.8,1 / 2 \mathrm{CH}_{2}\right), 6.17$ (dd, 1H, J 6.1, 7.7, CH-O), 7.17 (m, 5H, CH-Ar), 7.33 (m, 6H, $\mathrm{CH}$-Ar), 7.51 (dd, 1H, J 1.2, 2.1, CH-Ar), 7.90 (dt, 1H, J 1.4, 7.8, CH-Ar), 7.99 (td, 1H, J 1.6, 2.1, CH-Ar); ${ }^{13} \mathrm{C}$ NMR 43.1 $\left(\mathrm{CH}_{2}\right), 77.8$ (CH-O), 126.5 (2 CH-Ar), 126.7 (CH-Ar), 127.7 (CH-Ar), 128.1 (CH-Ar), $128.3 \quad(2$ CH-Ar), 128.4 (2 CH-Ar), 129.5 (2 CH-Ar), 129.6 (CH-Ar), 129.7 (CH-Ar), 132.1 ( $\left.C_{\text {quat }}\right), 132.9$ (CH-Ar), $134.5\left(C_{\text {quat }}\right), 136.7\left(C_{\text {quat }}\right), 139.7\left(C_{\text {quat }}\right), 164.4(C=\mathrm{O})$; HPLC: eluent hexane/iPr-OH/TFA 100:0.03:0.05, retention times $t_{R}-16.80$ and $t_{R}-27.12$ min.

1,2-Diphenylethyl 4-chlorobenzoate 9d: $80 \%$ yield; $\mathrm{R}_{\mathrm{f}}$ 0.49 (hexane:EtOAc 90:10); m. p. $100-101{ }^{\circ} \mathrm{C} ;{ }^{1} \mathrm{H}$ NMR 3.18 (A part of $\mathrm{ABX}, 1 \mathrm{H}, \mathrm{J}_{\mathrm{AX}} 6.0, \mathrm{~J}_{\mathrm{AB}} 13.8,1 / 2 \mathrm{CH}_{2}$ ), 3.34 (B part of $\left.\mathrm{ABX}, 1 \mathrm{H}, \mathrm{J}_{\mathrm{BX}} 7.6, \mathrm{~J}_{\mathrm{AB}} 13.8,1 / 2 \mathrm{CH}_{2}\right), 6.16(\mathrm{dd}$, 1H, J 6.0, 7.6, CH-O), 7.17 (m, 5H, CH-Ar), 7.32 (m, 5H, $\mathrm{CH}$-Ar), 7.39 (dd, 2H, J 0.6, 8.4, CH-Ar), 7.96 (dd, 2H, J 0.6, 8.4, CH-Ar); ${ }^{13} \mathrm{C}$ NMR $43.1\left(\mathrm{CH}_{2}\right), 77.5$ (CH-O), 126.5 (2 CH-Ar), 126.6 (CH-Ar), 128.91 (CH-Ar), 128.3 (2 CH-Ar), 128.4 (2 CH-Ar), 128.7 (2 CH-Ar), 128.8 
$\left(C_{\text {quat }}\right), 129.5$ (2 CH-Ar), 133.0 (2 CH-Ar), $136.8\left(C_{\text {quat }}\right)$, $139.4\left(C_{\text {quat }}\right), 139.8\left(C_{\text {quat }}\right), 164.8(C=\mathrm{O})$; HPLC: eluent hexane/iPr-OH/TFA 100:0.03:0.05, retention times $t_{R}-1$ 8.85 and $t_{R}-29.34$ min.

1,2-Diphenylethyl 2,4-dichlorobenzoate 9e: $75 \%$ yield; $\mathrm{R}_{\mathrm{f}} 0.42$ (hexane:EtOAc 90:10); ${ }^{1} \mathrm{H}$ NMR 3.18 (A part of $\mathrm{ABX}, 1 \mathrm{H}, \mathrm{J}_{\mathrm{AX}}$ 6.3, $\mathrm{J}_{\mathrm{AB}} 13.8,1 / 2 \mathrm{CH}_{2}$ ), 3.34 (B part of $\left.\mathrm{ABX}, 1 \mathrm{H}, \mathrm{J}_{\mathrm{BX}} 7.7, \mathrm{~J}_{\mathrm{AB}} 13.8,1 / 2 \mathrm{CH}_{2}\right), 6.19$ (dd, 1H, J 6.3, 7.7, $\mathrm{CH}-\mathrm{O}), 7.13$ (m, 2H, CH-Ar), 7.24 (m, 5H, CH-Ar), 7.33 (m, 4H, CH-Ar), 7.44 (d, 1H, J 2.0, CH-Ar), 7.69 (d, 1H, J 8.4, CH-Ar); ${ }^{13} \mathrm{C}$ NMR $43.0\left(\mathrm{CH}_{2}\right), 78.4$ (CH-O), 126.6 (CH-Ar), 126.7 (2 CH-Ar), 126.9 (CH-Ar), 128.2 (CH-Ar), 128.3 (2 CH-Ar), 128.4 (2 CH-Ar), 129.5 (2 CH-Ar), 131.0 (CH-Ar), 132.5 (CH-Ar), 134.9 ( $\left.C_{\text {quat }}\right)$, $136.6\left(C_{\text {quat }}\right), 138.2\left(C_{\text {quat }}\right), 139.4\left(C_{\text {quat }}\right), 163.8(C=\mathrm{O})$; HPLC: eluent hexane/iPr-OH/TFA 100:0.03:0.05, retention times $t_{R}-18.51$ and $t_{R}-29.09$ min.

1,2-Diphenylethyl 2-naphthoate 9f: 70\% yield; $\mathrm{R}_{\mathrm{f}} 0.67$ (hexane:EtOAc 85:15); m. p. 99-100 ${ }^{\circ} \mathrm{C} ;{ }^{1} \mathrm{H}$ NMR 3.24 (A part of $\mathrm{ABX}, 1 \mathrm{H}, \mathrm{J}_{\mathrm{AX}} 6.1, \mathrm{~J}_{\mathrm{AB}} 13.8,1 / 2 \mathrm{CH}_{2}$ ), 3.41 (B part of $\left.\mathrm{ABX}, 1 \mathrm{H}, \mathrm{J}_{\mathrm{BX}} 7.5, \mathrm{~J}_{\mathrm{AB}} 13.8,1 / 2 \mathrm{CH}_{2}\right), 6.25(\mathrm{dd}, 1 \mathrm{H}$, J 6.1, 7.5, CH-O), $7.18(\mathrm{~m}, 5 \mathrm{H}, \mathrm{CH}-\mathrm{Ar}), 7.34(\mathrm{~m}, 5 \mathrm{H}$, $\mathrm{CH}$-Ar), 7.41 (m, 2H, CH-Ar), 7.54 (tdd, 2H, J 1.6, 6.9, 12.3, CH-Ar), 7.85 (d, 2H, J 8.6, CH-Ar), 7.94 (dd, 1H, J 1.8, 7.7, CH-Ar), 8.05 (dd, 1H, J 1.6, 8.6, CH-Ar), 8.59 (s, $1 \mathrm{H}, \mathrm{CH}-\mathrm{Ar}) ;{ }^{13} \mathrm{C}$ NMR $43.2\left(\mathrm{CH}_{2}\right), 77.4(\mathrm{CH}-\mathrm{O})$, 125.2 (CH-Ar), 126.5 (2 CH-Ar), 126.6 (2 CH-Ar), 127.6 ( $\left.C_{\text {quat }}\right), 127.7$ (CH-Ar), 128.0 (CH-Ar), 128.1 (CH-Ar), 128.2 (CH-Ar), 128.3 (2 CH-Ar), 128.4 (2 CH-Ar), 129.4 (CH-Ar), 129.6 (2 CH-Ar), 131.1 $(C H-A r), 132.5\left(C_{\text {quat }}\right), 135.5\left(C_{\text {quat }}\right), 136.9\left(C_{\text {quat }}\right), 140.1$ $\left(C_{\text {quat }}\right), 165.8(C=\mathrm{O})$; HPLC: eluent hexane/iPr-OH/TFA 100:0.03:0.05, retention times $t_{R}-1 \quad 15.45$ and $t_{R}-216.49$ $\min$.

1-(2-Nitrophenyl)ethyl benzoate 10a [81]: $80 \%$ yield; $\mathrm{R}_{\mathrm{f}} 0.32$ (hexane: $\mathrm{CH}_{2} \mathrm{Cl}_{2}$ 50:50); ${ }^{1} \mathrm{H}$ NMR (600 MHz) 1.79 (d, 3H, J 6.5, $\left.\mathrm{CH}_{3}\right), 6.57$ (q, 1H, J 6.5, 12.9, CH-O), 7.43 (t, 1H, J 7.3, CH-Ar), 7.45 (t, 2H, J 7.8, CH-Ar), 7.58 (t, 1H, J 7.4, CH-Ar), 7.62 (t, 1H, J 7.4, CH-Ar), 7.74 (d, 1H, J 7.9, CH-Ar), 7.97 (d, 1H, J 8.2, CH-Ar), 8.07 (d, 2H, J 7.3, CH-Ar); ${ }^{13} \mathrm{C}$ NMR $22.1\left(\mathrm{CH}_{3}\right), 68.7$ (CH-O), 124.4 (CH-Ar), 127.1 (CH-Ar), 128.3 (CH-Ar), 128.4 (2 CH-Ar), 129.6 (2 CH-Ar), 129.8 ( $\left.C_{\text {quat }}\right), 133.2$ $(C \mathrm{H}-\mathrm{Ar}), 133.6$ (CH-Ar), $138.1\left(C_{\text {quat }}\right), 147.6\left(C_{\text {quat }}\right)$, $165.4 \quad(C=\mathrm{O})$; HPLC: eluent hexane/iPr-OH/TFA 100:0.1:0.05, retention times $t_{R}-136.51$ and $t_{R}-239.34$ $\min$.

1-(2-Nitrophenyl)ethyl 2-chlorobenzoate 10b: $82 \%$ yield; $\mathrm{R}_{\mathrm{f}} 0.30$ (hexane: $\mathrm{CH}_{2} \mathrm{Cl}_{2} 50: 50$ ); m. p. $64-65^{\circ} \mathrm{C} ;{ }^{1} \mathrm{H}$ NMR 1.79 (d, 3H, J 6.5, $\left.\mathrm{CH}_{3}\right), 6.62$ (q, 1H, J 6.5, 12.9, $\mathrm{CH}-\mathrm{O}), 7.433$ (m, 1H, CH-Ar), 7.44 (m, 3H, CH-Ar), 7.64 (tdd, 1H, J 0.4, 1.3, 7.8, CH-Ar), 7.79 (dd, 1H, J 1.5, 7.9, CH-Ar), 7.84 (ddd, 1H, J 0.6, 1.7, 7.6, CH-Ar), 7.98 (dd, 1H, J 1.3, 8.2, CH-Ar); ${ }^{13} \mathrm{C}$ NMR $22.1\left(\mathrm{CH}_{3}\right), 69.6$ (CH-O), 124.5 (CH-Ar), 126.6 (CH-Ar), 127.5 (CH-Ar), 128.5 (CH-Ar), 129.8 ( $\left.C_{\text {quat }}\right), 131.1$ (CH-Ar), 131.5
(CH-Ar), $131.6\left(C_{\text {quat }}\right), 132.7$ (CH-Ar), 133.7 (CH-Ar), $137.6\left(C_{\text {quat }}\right), 147.6\left(C_{\text {quat }}\right), 164.6(C=\mathrm{O})$; HPLC: eluent hexane/iPr-OH/TFA 100:0.1:0.05, retention times $t_{R}-1$ 25.35 and $t_{R}-227.34$ min.

1-(2-Nitrophenyl)ethyl 3-chlorobenzoate 10c: $86 \%$ yield; $\mathrm{R}_{\mathrm{f}} 0.48$ (hexane: $\mathrm{CH}_{2} \mathrm{Cl}_{2}$ 50:50); ${ }^{1} \mathrm{H}$ NMR 1.79 (d, 3H, J 6.5, $\left.\mathrm{CH}_{3}\right), 6.57$ (q, 1H, J 6.5, 12.9, CH-O), 7.39 (t, 1H, J 7.7, CH-Ar), 7.44 (ddd, 1H, J 1.6, 7.2, 8.2, CH-Ar), 7.54 (ddd, 1H, J 1.2, 2.2, 8.0, CH-Ar), 7.63 (td, 1H, J 1.3, 7.9, $\mathrm{CH}$-Ar), 7.70 (td, 1H, J 1.7, 7.9, CH-Ar), 7.95 (m, 2H, $\mathrm{CH}$-Ar), 8.02 (td, 1H, J 1.7, 2.0, CH-Ar); ${ }^{13} \mathrm{C}$ NMR 22.0 $\left(\mathrm{CH}_{3}\right), 69.2$ (CH-O), 124.5 (CH-Ar), 127.0 (CH-Ar), 127.7 (CH-Ar), 128.5 (CH-Ar), 129.6 (2 CH-Ar), 129.8 $(C \mathrm{H}-\mathrm{Ar}), 131.6\left(C_{\text {quat }}\right), 133.2(\mathrm{CH}-\mathrm{Ar}), 133.7$ (CH-Ar), $134.6\left(C_{\text {quat }}\right), 137.6\left(C_{\text {quat }}\right), 147.7\left(C_{\text {quat }}\right), 164.2(C=\mathrm{O})$; HPLC: eluent hexane/iPr-OH/TFA 100:0.1:0.05, retention times $t_{R}-124.22$ and $t_{R}-225.84$ min.

1-(2-Nitrophenyl)ethyl 4-chlorobenzoate 10d: $79 \%$ yield; $\mathrm{R}_{\mathrm{f}} 0.31$ (hexane: $\mathrm{CH}_{2} \mathrm{Cl}_{2}$ 50:50); ${ }^{1} \mathrm{H}$ NMR $(600$ $\mathrm{MHz}) 1.79$ (d, 3H, J 6.5, $\left.\mathrm{CH}_{3}\right), 6.56$ (q, 1H, J 6.5, 13.0, $\mathrm{CH}-\mathrm{O}$ ), 7.42 (d, 2H, J 8.5, CH-Ar), 7.45 (td, 1H, J 0.8, 8.2, CH-Ar), 7.63 (t, 1H, J 7.6, CH-Ar), 7.70 (d, 1H, J 7.6, CH-Ar), 7.97 (d, 1H, J 8.4, CH-Ar), 7.99 (d, 2H, J 8.5, $\mathrm{CH}$-Ar $) ;{ }^{13} \mathrm{C}$ NMR $22.1\left(\mathrm{CH}_{3}\right), 69.1$ ( $\left.\mathrm{CH}-\mathrm{O}\right), 124.6$ (CH-Ar), 127.1 (CH-Ar), 128.3 ( $\left.C_{\text {quat }}\right), 128.5$ (CH-Ar), 128.8 (2 CH-Ar), 131.2 (2 CH-Ar), 133.7 (CH-Ar), $137.8\left(C_{\text {quat }}\right), 139.7\left(C_{\text {quat }}\right), 147.7\left(C_{\text {quat }}\right), 164.6(C=\mathrm{O})$; HPLC: eluent hexane/iPr-OH/TFA 100:0.1:0.05, retention times $t_{R}-125.70$ and $t_{R}-227.46 \mathrm{~min}$.

1-(2-Nitrophenyl)ethyl 2,4-dichlorobenzoate 10e: $76 \%$ yield; $\mathrm{R}_{\mathrm{f}} 0.32$ (hexane: $\mathrm{CH}_{2} \mathrm{Cl}_{2}$ 50:50); mp: $69-71{ }^{\circ} \mathrm{C} ;{ }^{1} \mathrm{H}$ NMR 1.79 (d, 3H, J 6.5, $\left.\mathrm{CH}_{3}\right), 6.60$ (q, 1H, J 6.5, 12.9, $\mathrm{CH}-\mathrm{O}$ ), 7.31 (dd, 1H, J 2.0, 8.4, CH-Ar), 7.45 (ddd, 1H, J 1.5, 7.3. 8.2, CH-Ar), 7.48 (d, 1H, J 2.1, CH-Ar), 7.64 (ddd, 1H, J 1.2, 7.6, 8.0, CH-Ar), 7.75 (dd, 1H, J 1.5, 7.9, CH-Ar), 7.82 (d, 1H, J 8.4, CH-Ar), 7.98 (dd, 1H, J 1.2, 8.2, $\mathrm{CH}$-Ar); ${ }^{13} \mathrm{C}$ NMR $22.1\left(\mathrm{CH}_{3}\right), 69.9$ (CH-O), 124.6 (CH-Ar), 127.1 (CH-Ar), 127.4 (CH-Ar), 128.0 ( $\left.C_{\text {quat }}\right)$, 128.6 (CH-Ar), 131.1 (CH-Ar), 132.6 (CH-Ar), 133.7 $(C H-A r), 134.9\left(C_{\text {quat }}\right), 137.4\left(C_{\text {quat }}\right), 138.6\left(C_{\text {quat }}\right), 147.7$ $\left(C_{\text {quat }}\right), 163.7(C=\mathrm{O})$; HPLC: eluent hexane/iPr-OH/TFA 100:0.1:0.05, retention times $t_{R}-124.97$ and $t_{R}-226.44$ $\min$.

1-(2-Nitrophenyl)ethyl 2-naphthoate 10f: 70\% yield; $\mathrm{R}_{\mathrm{f}} 0.34$ (hexane: $\mathrm{CH}_{2} \mathrm{Cl}_{2}$ 50:50); m. p. $80-82{ }^{\circ} \mathrm{C} ;{ }^{1} \mathrm{H}$ NMR 1.84 (d, 3H, J 6.5, $\mathrm{CH}_{3}$ ), 6.64 (q, 1H, J 6.5, 12.9, CH-O), 7.42 (ddd, 1H, J 1.5, 7.4, 8.2, CH-Ar), $7.58(\mathrm{~m}, 3 \mathrm{H}$, CH-Ar), 7.78 (dd, 1H, J 1.5, 7.9, CH-Ar), 7.88 (m, 2H, $\mathrm{CH}$-Ar), 7.96 (m, 1H, CH-Ar), 7.97 (dd, 1H, J 1.4, 8.2, $\mathrm{CH}$-Ar), 8.06 (dd, 12H, J 1.7, 8.6, CH-Ar), 8.62 (s, 1H, $\mathrm{CH}$-Ar); ${ }^{13} \mathrm{C}$ NMR $22.1 \quad\left(\mathrm{CH}_{3}\right), 68.9$ (CH-O), 124.5 (CH-Ar), 125.1 (CH-Ar), 126.7 (CH-Ar), 127.1 (CH-Ar), 127.8 (CH-Ar), 128.2 (CH-Ar), 128.4 (2 CH-Ar), 129.3 $(C H-A r), 129.9\left(C_{\text {quat }}\right), 131.2(C H-A r), 132.4\left(C_{\text {quat }}\right)$, 133.6 (CH-Ar), $135.6\left(C_{\text {quat }}\right), 138.1\left(C_{\text {quat }}\right), 147.8\left(C_{\text {quat }}\right)$, $165.6(C=\mathrm{O})$; HPLC: eluent hexane/iPr-OH/TFA 
100:0.1:0.05, retention times $t_{R}-152.59$ and $t_{R}-256.85$ $\min$.

1-(4-Nitrophenyl)ethyl benzoate 11a [82]: $82 \%$ yield; $\mathrm{R}_{\mathrm{f}}$ 0.40 (hexane: $\mathrm{CH}_{2} \mathrm{Cl}_{2} 40: 60$ ); m. p. $94-95^{\circ} \mathrm{C}$ (lit. [82] 94.8-95.5 ${ }^{\circ} \mathrm{C}$ ); ${ }^{1} \mathrm{H}$ NMR 1.70 (d, 3H, J 6.7, $\mathrm{CH}_{3}$ ), 6.18 (q, 1H, J 6.6, 13.2, CH-O), 7.46 (m, 2H, CH-Ar), 7.59 (m, $3 \mathrm{H}, \mathrm{CH}$-Ar), 8.09 (dt, 2H, J 1.4, 7.0, CH-Ar), 8.23 (dt, $2 \mathrm{H}, \mathrm{J} 1.9,8.8, \mathrm{CH}-\mathrm{Ar}) ;{ }^{13} \mathrm{C}$ NMR $22.4\left(\mathrm{CH}_{3}\right), 71.8$ (CH-O), 124.0 (2 CH-Ar), 126.7 (2 CH-Ar), 128.5 (2 CH-Ar), 129.7 (2 CH-Ar), 129.9 ( $\left.C_{\text {quat }}\right), 133.3$ (CH-Ar), $147.5\left(C_{\text {quat }}\right), 149.1\left(C_{\text {quat }}\right), 165.6(C=\mathrm{O})$; HPLC: eluent hexane/iPr-OH/TFA 100:0.1:0.05, retention times $t_{R}-1$ 43.59 and $t_{R}-246.09$ min.

1-(4-Nitrophenyl)ethyl 2-chlorobenzoate $11 \mathrm{~b}: 66 \%$ yield; $\mathrm{R}_{\mathrm{f}} 0.60$ (hexane: $\mathrm{CH}_{2} \mathrm{Cl}_{2} 40: 60$ ); m. p. $52-53{ }^{\circ} \mathrm{C} ;{ }^{1} \mathrm{H}$ NMR 1.71 (d, 3H, J 6.6, $\mathrm{CH}_{3}$ ), 6.19 (q, 1H, J 6.6, 13.3, CH-O), 7.33 (m, 1H, CH-Ar), 7.45 (m, 2H, CH-Ar), 7.62 (dt, 2H, J 1.7, 8.6, CH-Ar), 7.85 (ddd, 1H, J 0.5, 1.6, 8.6, CH-Ar), 8.23 (dt, 2H, J 2.0, 8.8, CH-Ar); ${ }^{13} \mathrm{C} \mathrm{NMR} 22.3\left(\mathrm{CH}_{3}\right)$, 72.7 (CH-O), 123.9 (2 CH-Ar), 126.7 (CH-Ar), 126.9 (2 CH-Ar), 129.7 ( $\left.C_{\text {quat }}\right), 131.2$ (CH-Ar), 131.4 (CH-Ar), $132.8(C \mathrm{H}-\mathrm{Ar}), 133.8\left(C_{\text {quat }}\right), 147.6\left(C_{\text {quat }}\right), 148.5\left(C_{\text {quat }}\right)$, $164.7(C=\mathrm{O})$; HPLC: eluent hexane/iPr-OH/TFA 100:0.1: 0.05 , retention times $t_{R}-124.86$ and $t_{R}-225.85 \mathrm{~min}$.

1-(4-Nitrophenyl)ethyl 3-chlorobenzoate 11c: $88 \%$ yield; $\mathrm{R}_{\mathrm{f}} 0.53$ (hexane: $\mathrm{CH}_{2} \mathrm{Cl}_{2} 40: 60$ ); m. p. $54-55^{\circ} \mathrm{C} ;{ }^{1} \mathrm{H}$ NMR 1.71 (d, 3H, J 6.6, $\left.\mathrm{CH}_{3}\right), 6.17$ (q, 1H, J 6.6, 13.3, CH-O), 7.40 (t, 1H, J 7.9, CH-Ar), 7.55 (ddd, 1H, J 1.2, 2.2, 8.1, CH-Ar), 7.59 (dt, 2H, J 2.2, 8.8, CH-Ar), 7.96 (dt, 1H, J 1.4, 7.7, CH-Ar), 8.04 (dd, 1H, J 1.7, 2.0, CH-Ar), 8.23 (dt, 2H, J 2.2, 8.8, CH-Ar); ${ }^{13} \mathrm{C}$ NMR $22.2\left(\mathrm{CH}_{3}\right), 72.3$ (CH-O), 123.9 (2 CH-Ar), 126.7 (2 CH-Ar), 127.8 (CH-Ar), 129.6 (CH-Ar), 129.8 (CH-Ar), $131.6\left(C_{\text {quat }}\right)$, $133.3(C \mathrm{H}-\mathrm{Ar}), 134.6\left(C_{\text {quat }}\right), 147.6\left(C_{\text {quat }}\right), 148.6\left(C_{\text {quat }}\right)$, $164.3(C=\mathrm{O})$; HPLC: eluent hexane/iPr-OH/TFA 100:0.1: 0.05 , retention times $t_{R}-121.62$ and $t_{R}-222.50$ min.

1-(4-Nitrophenyl)ethyl 4-chlorobenzoate 11d: $64 \%$ yield, $\mathrm{R}_{\mathrm{f}} 0.50$ (hexane: $\mathrm{CH}_{2} \mathrm{Cl}_{2} 40: 60$ ); m. p. $61-63{ }^{\circ} \mathrm{C} ;{ }^{1} \mathrm{H}$ NMR 1.70 (d, 3H, J 6.6, $\mathrm{CH}_{3}$ ), 6.16 (q, 1H, J 6.6, 13.3, $\mathrm{CH}$-O), 7.43 (dt, 2H, J 2.2, 8.7, CH-Ar), 7.59 (dt, 2H, J 2.2, 8.7, CH-Ar), 8.01 (dt, 2H, J 2.2, 8.7, CH-Ar), 8.23 (dt, 2H, J 2.2, 8.7, CH-Ar); ${ }^{13} \mathrm{C}$ NMR $22.3\left(\mathrm{CH}_{3}\right), 72.2$ (CH-O), 124.0 (2 CH-Ar), 126.8 (2 CH-Ar), 128.3 ( $\left.C_{\text {quat }}\right)$, 128.9 (2 CH-Ar), 131.0 (2 CH-Ar), $139.9\left(C_{\text {quat }}\right), 147.6$ $\left(C_{\text {quat }}\right), 148.8\left(C_{\text {quat }}\right), 164.8(C=\mathrm{O})$; HPLC: eluent hexane/iPr-OH/TFA 100:0.1:0.05, retention times $t_{R}-121.71$ and $t_{R}-222.66 \mathrm{~min}$.

1-(4-Nitrophenyl)ethyl 2,4-dichlorobenzoate 11e: $22 \%$ yield; $\mathrm{R}_{\mathrm{f}} 0.63$ (hexane: $\mathrm{CH}_{2} \mathrm{Cl}_{2} 60: 40$ ); m. p. $111-113^{\circ} \mathrm{C}$; ${ }^{1} \mathrm{H}$ NMR 1.71 (d, 3H, J 6.6, $\left.\mathrm{CH}_{3}\right), 6.17$ (q, 1H, J 6.6, 13.2, $\mathrm{CH}-\mathrm{O}$ ), 7.32 (dd, 1H, J 2.0, 8.4, CH-Ar), 7.49 (d, 1H, J 2.0, CH-Ar), 7.61 (dt, 2H, J 2.2, 8.7, CH-Ar), 7.83 (d, 1H, J 8.4, CH-Ar), 8.23 (dt, 2H, J 2.2, 8.7, CH-Ar); ${ }^{13} \mathrm{C}$ NMR $22.2\left(\mathrm{CH}_{3}\right), 73.0$ (CH-O), 123.9 (2 $\left.\mathrm{CH}-\mathrm{Ar}\right), 126.9$ (2 CH-Ar), 127.1 (CH-Ar), 127.9 ( $\left.C_{\text {quat }}\right), 131.1$ (CH-Ar),
132.6 (CH-Ar), $135.0\left(C_{\text {quat }}\right), 138.7\left(C_{\text {quat }}\right), 147.6\left(C_{\text {quat }}\right)$, $148.2\left(C_{\text {quat }}\right), 163.8(C=\mathrm{O})$; HPLC: eluent hexane/iPr-OH/ TFA 100:0.1:0.05, retention times $t_{R}-123.85$ and $t_{R}-2$ $24.70 \mathrm{~min}$.

1-(4-Nitrophenyl)ethyl 2-naphthoate 11f: $17 \%$ yield; $\mathrm{R}_{\mathrm{f}}$ 0.54 (hexane: $\mathrm{CH}_{2} \mathrm{Cl}_{2}$ 40:60); m. p. $65-66^{\circ} \mathrm{C}$; ${ }^{1} \mathrm{H}$ NMR 1.74 (d, 3H, J 6.6, $\mathrm{CH}_{3}$ ), 6.23 (q, 1H, J 6.6, 13.3, CH-O), 7.57 (m, 2H, CH-Ar), 7.62 (dt, 2H, J 2.2, 8.9, CH-Ar), 7.88 (m, 2H, CH-Ar), 7.96 (ddd, 1H, J 0.6, 1.6, 7.6, $\mathrm{CH}$-Ar), 8.08 (dd, 1H, J 1.7, 8.6, CH-Ar), 8.22 (dt, 2H, J $2.1,8.9, \mathrm{CH}-\mathrm{Ar}), 8.64$ (s, $1 \mathrm{H}, \mathrm{CH}-\mathrm{Ar}) ;{ }^{13} \mathrm{C} \mathrm{NMR} 22.3$ $\left(\mathrm{CH}_{3}\right), 71.9$ (CH-O), 123.9 (2 $\left.\mathrm{CH}-\mathrm{Ar}\right), 125.0$ (CH-Ar), 126.7 (2 CH-Ar), 126.8 (CH-Ar), 127.0 ( $\left.C_{\text {quat }}\right), 127.8$ (CH-Ar), 128.3 (CH-Ar), 128.4 (CH-Ar), 129.3 (CH-Ar), 131.2 (CH-Ar), $132.4\left(C_{\text {quat }}\right), 135.6\left(C_{\text {quat }}\right), 147.5\left(C_{\text {quat }}\right)$, $149.1\left(C_{\text {quat }}\right), 165.7(C=\mathrm{O})$; HPLC: eluent hexane/iPr-OH/ TFA 100:0.1:0.05, retention times $t_{R}-140.97$ and $t_{R}-2$ $43.40 \mathrm{~min}$.

1-(2-Methoxyphenyl)propyl benzoate 12a: $66 \%$ yield; $\mathrm{R}_{\mathrm{f}} 0.46$ (hexane: $\mathrm{CH}_{2} \mathrm{Cl}_{2}$ 60:40); ${ }^{1} \mathrm{H}$ NMR $(600 \mathrm{MHz})$ 0.98 (t, 3H, J 7.4, $\left.\mathrm{CH}_{3}\right), 1.97\left(\mathrm{~m}, 2 \mathrm{H}, \mathrm{CH}_{2}\right), 3.85$ (s, 3H, $\left.\mathrm{OCH}_{3}\right), 6.37$ (t, 1H, J 6.4, CH-O), 6.88 (d, 1H, J 8.2, $\mathrm{CH}$-Ar), 6.93 (t, 1H, J 7.4, CH-Ar), 7.23 (ddd, 1H, J 1.5, 7.8, 8.3, CH-Ar), 7.39 (dd, 1H, J 1.4, 7.6, CH-Ar), 7.44 (t, 2H, J 7.6, CH-Ar), 7.54 (tt, 1H, J 1.4, 7.4, CH-Ar), 8.11 (dd, 2H, J 1.2, 8.4, CH-Ar); ${ }^{13} \mathrm{C}$ NMR $9.9\left(\mathrm{CH}_{3}\right), 28.6$ $\left(\mathrm{CH}_{2}\right), 55.5\left(\mathrm{OCH}_{3}\right), 72.3(\mathrm{CH}-\mathrm{O}), 110.5(\mathrm{CH}-\mathrm{Ar}), 120.5$ (CH-Ar), 126.1 (CH-Ar), 128.3 (2 CH-Ar), 128.5 $(C H-A r), 129.4\left(C_{\text {quat }}\right), 129.6$ (2 $\left.C H-A r\right), 130.7\left(C_{\text {quat }}\right)$, $132.8(C \mathrm{H}-\mathrm{Ar}), 156.2\left(C_{\text {quat }}\right), 165.8(C=\mathrm{O})$; HPLC: eluent hexane/iPr-OH/TFA 100:0.5:0.05, retention times $t_{R}-15.55$ and $t_{R}-26.21$ min.

1-(2-Methoxyphenyl)propyl 2-chlorobenzoate 12b: 99 $\%$ yield; $\mathrm{R}_{\mathrm{f}} 0.20$ (hexane: $\left.\mathrm{CH}_{2} \mathrm{Cl}_{2} 70: 30\right) ;{ }^{1} \mathrm{H}$ NMR (600 $\mathrm{MHz}) 0.99$ (t, 3H, J 7.4, $\left.\mathrm{CH}_{3}\right), 1.98\left(\mathrm{~m}, 2 \mathrm{H}, \mathrm{CH}_{2}\right), 3.86$ (s, $\left.3 \mathrm{H}, \mathrm{OCH}_{3}\right), 6.40$ (t, 1H, J 6.4, CH-O), 6.88 (d, 1H, J 8.2, CH-Ar), 6.94 (t, 1H, J 7.4, CH-Ar), 7.25 (ddd, 1H, J 1.4, 7.2, 8.7, CH-Ar), 7.30 (dd, 1H, J 1.1, 7.4, CH-Ar), 7.41 (m, 3H, CH-Ar), 7.864 (dd, 1H, J 1.5, 7.7, CH-Ar); ${ }^{13} \mathrm{C}$ NMR $9.9\left(\mathrm{CH}_{3}\right), 28.4\left(\mathrm{CH}_{2}\right), 55.5\left(\mathrm{OCH}_{3}\right), 73.2(\mathrm{CH}-\mathrm{O})$, 110.5 (CH-Ar), 120.5 (CH-Ar), 126.5 (2 CH-Ar), 128.6 $(C H-A r), 128.9\left(C_{\text {quat }}\right), 130.6\left(C_{\text {quat }}\right), 131.0(C \mathrm{H}-\mathrm{Ar})$, 131.4 (CH-Ar), 132.3 (CH-Ar), 133.6 ( $\left.C_{\text {quat }}\right), 156.3$ $\left(C_{\text {quat }}\right), 165.0(C=\mathrm{O})$; HPLC: eluent hexane/iPr-OH/TFA 100:0.5:0.05, retention times $t_{R}-17.20$ and $t_{R}-27.92 \mathrm{~min}$.

1-(2-Methoxyphenyl)propyl 3-chlorobenzoate 12c: 95 \% yield; $\mathrm{R}_{\mathrm{f}} 0.11$ (hexane: $\left.\mathrm{CH}_{2} \mathrm{Cl}_{2} 70: 630\right) ;{ }^{1} \mathrm{H}$ NMR (600 $\mathrm{MHz}) 0.98$ (t, 3H, J 7.4, $\left.\mathrm{CH}_{3}\right), 1.98\left(\mathrm{~m}, 2 \mathrm{H}, \mathrm{CH}_{2}\right), 3.86$ (s, $\left.3 \mathrm{H}, \mathrm{OCH}_{3}\right), 6.36$ (t, 1H, J 6.4, CH-O), 6.88 (d, 1H, J 8.3, $\mathrm{CH}$-Ar), 6.94 (t, 1H, J 7.5, CH-Ar), 7.25 (ddd, 1H, J 1.0, 7.3, 8.2, CH-Ar), 7.37 (m, 2H, CH-Ar), 7.51 (dt, 1H, J 0.8, 8.0, CH-Ar), 7.98 (d, 1H, J 7.7, CH-Ar), 8.07 (s, 1H, $\mathrm{CH}-\mathrm{Ar}) ;{ }^{13} \mathrm{C} \mathrm{NMR} 9.9\left(\mathrm{CH}_{3}\right), 28.5\left(\mathrm{CH}_{2}\right), 55.5\left(\mathrm{OCH}_{3}\right)$, 72.9 (CH-O), 110.6 (CH-Ar), 120.6 (CH-Ar), 126.2 (CH-Ar), 127.8 (CH-Ar), 128.7 (CH-Ar), 129.0 ( $\left.C_{\text {quat }}\right)$, 129.6 (CH-Ar), 129.7 (CH-Ar), 132.5 ( $\left.C_{\text {quat }}\right), 132.8$ 
$(C H-A r), 134.4\left(C_{\text {quat }}\right), 156.2\left(C_{\text {quat }}\right), 165.6(C=\mathrm{O})$; HPLC: eluent hexane/iPr-OH/TFA 100:0.5:0.05, retention times $t_{R}-14.69$ and $t_{R}-25.15$ min.

1-(2-Methoxyphenyl)propyl 4-chlorobenzoate 12d: 99\% yield; $\mathrm{R}_{\mathrm{f}} 0.19$ (hexane: $\mathrm{CH}_{2} \mathrm{Cl}_{2} 70: 30$ ); m. p. $169-170^{\circ} \mathrm{C}$; ${ }^{1} \mathrm{H}$ NMR (600 MHz) 0.97 (t, 3H, J 7.4, $\left.\mathrm{CH}_{3}\right), 1.98$ (m, $\left.2 \mathrm{H}, \mathrm{CH}_{2}\right), 3.86$ (s, 3H, OCH $\left.\mathrm{O}_{3}\right), 6.35$ (t, 1 H, J 6.4, $\mathrm{CH}-\mathrm{O}$ ), 6.88 (d, 1H, J 8.2, CH-Ar), 6.94 (t, 1H, J 7.5, CH-Ar), 7.24 (ddd, $1 \mathrm{H}, \mathrm{J} 1.5,7.6,8.3, \mathrm{CH}-\mathrm{Ar}$ ), 7.36 (dd, 1H, J 1.2, 7.5, CH-Ar), 7.41 (d, 2H, J 8.5, CH-Ar), 8.041 (d, 2H, J 8.5, CH-Ar); ${ }^{13} \mathrm{C}$ NMR $9.9\left(\mathrm{CH}_{3}\right), 28.5\left(\mathrm{CH}_{2}\right), 55.5$ $\left(\mathrm{OCH}_{3}\right), 72.6(\mathrm{CH}-\mathrm{O}), 126.2(\mathrm{CH}-\mathrm{Ar}), 128.6(\mathrm{CH}-\mathrm{Ar})$, 128.7 (2 CH-Ar), 129.1 ( $\left.C_{\text {quat }}\right), 129.2\left(C_{\text {quat }}\right), 129.4$ (CH-Ar), 131.0 (2 CH-Ar), 131.9 (CH-Ar), 139.2 ( $C_{\text {quat }}$ ), $156.2\left(C_{\text {quat }}\right), 164.9(C=\mathrm{O})$; HPLC: eluent hexane/iPr-OH/ TFA 100:0.5:0.05, retention times $t_{R}-14.71$ and $t_{R}-25.22$ min.

1-(2-Methoxyphenyl)propyl 2,4-dichlorobenzoate 12e: $99 \%$ yield; $\mathrm{R}_{\mathrm{f}} 0.27$ (hexane: $\mathrm{CH}_{2} \mathrm{Cl}_{2}$ 70:30); ${ }^{1} \mathrm{H}$ NMR $(600 \mathrm{MHz}) 0.98\left(\mathrm{t}, 3 \mathrm{H}, \mathrm{J}\right.$ 7.4, $\left.\mathrm{CH}_{3}\right), 1.97\left(\mathrm{~m}, 2 \mathrm{H}, \mathrm{CH}_{2}\right)$, $3.86\left(\mathrm{~s}, 3 \mathrm{H}, \mathrm{OCH}_{3}\right), 6.38$ (t, 1H, J 6.4, $\left.\mathrm{CH}-\mathrm{O}\right), 6.89$ (d, $1 \mathrm{H}, \mathrm{J}$ 8.2, CH-Ar), 6.95 (t, 1H, J 7.5, CH-Ar), 7.26 (ddd, $1 \mathrm{H}, \mathrm{J}$ 1.57, 7.6, 8.1, CH-Ar), 7.29 (dd, 1H, J 2.0, 8.4, $\mathrm{CH}-\mathrm{Ar}$ ), 7.38 (dd, 1H, J 1.6, 7.6, CH-Ar), 7.47 (d, 1H, J 2.0, CH-Ar), 7.84(d, $1 \mathrm{H}, \mathrm{J} 8.4, \mathrm{CH}-\mathrm{Ar}) ;{ }^{13} \mathrm{C}$ NMR 9.9 $\left(\mathrm{CH}_{3}\right), 28.4\left(\mathrm{CH}_{2}\right), 55.5\left(\mathrm{OCH}_{3}\right), 72.5(\mathrm{CH}-\mathrm{O}), 110.5$ (CH-Ar), 120.5 (CH-Ar), 126.5 (CH-Ar), 127.0 (CH-Ar), $128.6\left(C_{\text {quat }}\right), 128.7$ (CH-Ar), 128.9 (C $\left.C_{\text {quat }}\right), 131.0$ $(\mathrm{CH}-\mathrm{Ar}), 132.6(\mathrm{CH}-\mathrm{Ar}), 134.8\left(C_{\text {quat }}\right), 138.1\left(C_{\text {quat }}\right)$, $156.3\left(C_{\text {quat }}\right), 164.1 \quad(C=\mathrm{O})$; HPLC: eluent hexane/iPr-OH/TFA 100:0.5:0.05, retention times $t_{R}-14.79$ and $\mathrm{t}_{\mathrm{R}}-25.23 \mathrm{~min}$.

1-(2-Methoxyphenyl)propyl 2-naphthoate 12f: 94\% yield; $\mathrm{R}_{\mathrm{f}} 0.22$ (hexane: $\mathrm{CH}_{2} \mathrm{Cl}_{2} 70: 30$ ); m. p. $73-75^{\circ} \mathrm{C} ;{ }^{1} \mathrm{H}$ NMR $(600 \mathrm{MHz}) 1.02\left(\mathrm{t}, 3 \mathrm{H}, \mathrm{J} 7.4, \mathrm{CH}_{3}\right), 2.02\left(\mathrm{~m}, 2 \mathrm{H}, \mathrm{CH}_{2}\right)$, 3.85 (s, 3H, OCH $\left.H_{3}\right), 6.44$ (t, 1H, J 6.4, CH-O), 6.87 (d, 1H, J 8.3, CH-Ar), 6.94 (t, 1H, J 7.5, CH-Ar), 7.24 (ddd, $1 \mathrm{H}, \mathrm{J}$ 1.6, 7.8, 8.8, CH-Ar), $7.46(\mathrm{dd}, 1 \mathrm{H}, \mathrm{J}$ 1.6, 7.6, $\mathrm{CH}-\mathrm{Ar}), 7.51$ (td, $1 \mathrm{H}, \mathrm{J} 1.6,8.2, \mathrm{CH}-\mathrm{Ar}), 7.55(\mathrm{td}, 1 \mathrm{H}, \mathrm{J}$ 1.1, 8.1, CH-Ar), 7.86 (t, 2H, J 9.0, CH-Ar), $7.95(\mathrm{~d}, 1 \mathrm{H}$, J 8.1, CH-Ar), 8.13 (dd, 1H, J 1.6, 8.6, CH-Ar), 8.67 (s, $1 \mathrm{H}, \mathrm{CH}-\mathrm{Ar}) ;{ }^{13} \mathrm{C}$ NMR $10.0\left(\mathrm{CH}_{3}\right), 28.6\left(\mathrm{CH}_{2}\right), 55.5$ $\left(\mathrm{OCH}_{3}\right), 72.4$ (CH-O), 110.5 (CH-Ar), 120.5 (CH-Ar), 125.3 (CH-Ar), 126.2 (CH-Ar), 126.6 (CH-Ar), 127.7 (CH-Ar), $128.0\left(C_{\text {quat }}\right), 128.1$ (CH-Ar), $128.2(\mathrm{CH}-\mathrm{Ar})$, 128.5 (CH-Ar), 129.3 (CH-Ar), $129.4\left(C_{\text {quat }}\right), 131.0$ $(C \mathrm{H}-\mathrm{Ar}), 132.5\left(C_{\text {quat }}\right), 135.5\left(C_{\text {quat }}\right), 156.2\left(C_{\text {quat }}\right), 165.9$ $(C=\mathrm{O})$; HPLC: eluent hexane/iPr-OH/TFA 100:0.5:0.05, retention times $t_{R}-17.95$ and $t_{R}-29.06$ min.

\section{Acknowledgements}

We thank Irena Zagranyarska and Dr. Kalina Kostova for supplying us with a sample of 1-(2-methoxyphenyl)propanol. The financial support by the National Research
Fund of Bulgaria for the purchase of Bruker Avance II+ 600 NMR spectrometer, Project UNA-17/2005, is also gratefully acknowledged.

\section{References}

[1] R. Noyori and M. Kitamura, "Enantioselective Addition of Organometallic Reagents to Carbonyl Compounds: Chirality Transfer, Multiplication, and Amplification," Angewandte Chemie International Edition, Vol. 30, No. 1, January 1991, pp. 49-69.

[2] K. Soai and S. Niwa, "Enantioselective Addition of Organozinc Reagents to Aldehydes," Chemical Reviews, Vol. 92, No. 5, July 1992, pp. 833-856.

[3] R. Noyori, "Asymmetric Catalysis in Organic Synthesis," In: R. Noyori, Ed., Wiley, New York, 1994.

[4] L. Pu and H. B. Yu, "Catalytic Asymmetric Organozinc Additions to Carbonyl Compounds," Chemical Reviews, Vol. 101, No. 3, March 2001, pp. 757-824.

[5] L. Pu, "Asymmetric Alkynylzinc Additions to Aldehydes and Ketones," Tetrahedron, Vol. 59, No. 50, December 2003, pp. 9873-9886.

[6] P. J. Walsh, "Titanium-Catalyzed Enantioselective Additions of Alkyl Groups to Aldehydes: Mechanistic Studies and New Concepts in Asymmetric Catalysis," Accounts of Chemical Research, Vol. 36, No. 10, October 2003, pp. 739-749.

[7] S.-H. Hsieh and H.-M. Gau, "Enantioselective Addition of Diethylzinc to Aldehydes Catalyzed by Titanium(IV) Complexes of $n$-Sulfonylated $\beta$-Amino Alcohols with Four Stereogenic Centers," Chirality, Vol. 18, No. 8, 2006, pp. 569-574.

[8] H. Y. Aboul-Enein and I. Ali, "Chiral Separations by Liquid Chromatography and Related Technologies," Marcel Dekker, New York, 2003.

[9] G. Gübitz and M. G. Schmid, "Chiral Separations: Methods and Protocols," Methods in Molecular Biology, Vol. 243, Humana Press, Totowa, 2004.

[10] G. Cox, "Preparative Enantioselective Chromatography," In: G. Cox, Ed., Blackwell Publishing, Oxford, 2005.

[11] T. J. Ward, "Chiral Separations," Analitical Chemistry, Vol. 78, No. 12, June 2006, pp. 3947-3956.

[12] G. Subramanian, Ed., "Chiral Separation Techniques: A Practical Approach," 3rd Edition, In: G. Subramanian, Ed., Wiley-VCH, Weinheim, 2007.

[13] Y. Okamoto and T. Ikai, "Chiral HPLC for Efficient Resolution of Enantiomers," Chemical Society Reviews, Vol. 37, No. 12, December 2008, pp. 2593-2608.

[14] W. H. Pirkle, D. W. House and J. M. Finn, "Broad Spectrum Resolution of Optical Isomers Using Chiral HPLC Bonded Phases," Journal of Chromatography A, Vol. 192, No. 1, April 1980, pp. 143-158.

[15] W. H. Pirkle, J. M. Finn, J. L. Schreiner and B. C. J. Hamper, "A Widely Useful Chiral Stationary Phase for the High-Performance Liquid-Chromatography Separation of Enantiomers," Journal of the American Chemical 
Society, Vol. 103, No. 13, July 1981, pp. 3964-3966.

[16] W. H. Pirkle and T. C. Pochapsky, "Considerations of Chiral Recognition Relevant to the Liquid Chromatography Separation of Enantiomers," Chemical Reviews, Vol. 89, No. 2, March 1989, pp. 347-362.

[17] I. W. Kim, J. K. Ryu, S. D. Ahn, J. H. Park, K.-P. Lee, J. J. Ryoo, M. H. Hyun, Y. Okamoto, C. Yamamoto and P. W. Carr, "Comparison of Chiral Separation on Amylose and Cellulose Tris(3,5-Dimethylphenylcarbamate)-Coated Zirconia in HPLC," Bulletin of the Korean Chemical Society, Vol. 24, No. 2, February 2003, pp. 239-242.

[18] B. Chankvetadze, T. Ikai, C. Yamamoto and Y. Okamoto, "High-Performance Liquid Chromatographic Enantioseparations on Monolithic Silica Columns Containing a Covalently Attached 3,5-Dimethylphenylcarbamate Derivative of Cellulose," Journal of Chromatography A, Vol. 1042, No. 1-2, July 2004, pp. 55-60.

[19] J. Bugla, I. Wandzik and W. Szeja, "High-Performance Liquid Chromatography Separation of Glycerol Derivatives on a Chiral Stationary Phase," Acta Chromatographica, No. 15, 2005, pp. 173-182.

[20] S. H. Kwon, Y. Okamoto, C. Yamamoto, W. Cheong, M. Moon and J. H. Park, "Cellulose Dimethylphenylcarbamate-Bonded Carbon-Clad Zirconia for Chiral Separation in High Performance Liquid Chromatography," Analytical Sciences, Vol. 22, No. 12, December 2006, pp. 1525-1529.

[21] S. Ottiger, J. Kluge, A. Rajendran and M. Mazzotti, "Enantioseparation of 1-Phenyl-1-Propanol on Cellulose-Derived Chiral Stationary Phase by Supercritical Fluid Chromatography II. Non-Linear Isotherm," Journal of Chromatography A, Vol. 1162, No. 1, August 2007, pp. 74-82.

[22] Y. Shimada, Y. Miyake, H. Matsuzawa and Y. Nishibayashi, "Ruthenium-Catalyzed Sequential Reactions: Deracemization of Secondary Benzylic Alcohols," Chemistry-An Asian Journal, Vol. 2, No. 3, March 2007, pp. 393-396.

[23] X. Chen, C. Yamamoto and Y. Okamoto, "Polysaccharide Derivatives as Useful Chiral Stationary Phases in High-Performance Liquid Chromatography," Pure and Applied Chemistry, Vol. 79, No. 9, July 2007, pp. 15611573.

[24] P. Sun, C. Wang, Z. S. Breitbach, Y. Zhang and D. W. Armstrong, "Development of New HPLC Chiral Stationary Phases Based on Native and Derivatized Cyclofructans," Analytical Chemistry, Vol. 81, No. 24, December 2009, pp. 10215-10226.

[25] M. Szaleniec, A. Dudzik, M. Pawul and B. Kozik, "Quantitative Structure Enantioselective Retention Relationship for High-Performance Liquid Chromatography Chiral Separation of 1-Phenylethanol Derivatives," Journal of Chromatography A, Vol. 1216, No. 34, August 2009, pp. 6224-6235.

[26] X.-H. Lai and S.-C. Ng, "Preparation and Chiral Recognition of a Novel Chiral Stationary Phase for High-Performance Liquid Chromatography, Based on Mono(6A- $N$-Allylamino-6A-Deoxy)-Perfunctionalized
Cyclodextrin and Covalently Bonded Silica Gel," Journal of Chromatography A, Vol. 1031, No. 1-2, March 2004, pp. 135-142.

[27] Q. Zhong, L. He, T. E. Beesley, W. S. Trahanovsky, P. Sun, C. Wang and D. W. Armstrong, "Development of Dinitrophenylated Cyclodextrin Derivatives for Enhanced Enantiomeric Separations by High-Performance Liquid Chromatography," Journal of Chromatography A, Vol. 1115, No. 1-2, May 2006, pp. 19-45.

[28] Y.-F. Poon, I. W. Muderawan and S.-C. Ng, "Synthesis and Application of Mono-2A-Azido- $2 A$-Deoxy- perphenylcarbamoylated-Cyclodextrin and Mono-2A-Azido$2 A$-Deoxyperacetylated Cyclodextrin as Chiral Stationary Phases for High-Performance Liquid Chromatography," Journal of Chromatography A, Vol. 1101, No. 1-2, January 2006, pp. 185-197.

[29] T.-T. Ong, R.-Q. Wang, I. W. Muderawan and S.-C. Ng, "Synthesis and Application of Mono-6-(3-Methylimidazolium)-6-Deoxyperphenylcarbamoyl-Cyclodextrin Chloride as Chiral Stationary Phases for High-Performance Liquid Chromatography and Supercritical Fluid Chromatography," Journal of Chromatography A, vol. 1182, no. 1, February 2008, pp. 136-140.

[30] R.-Q. Wang, T.-T. Ong and S.-C. Ng, "Synthesis of Cationic Cyclodextrin Derivatives and their Applications as Chiral Stationary Phases for High-Performance Liquid Chromatography and Supercritical Fluid Chromatography," Journal of Chromatography A, Vol. 1203, No. 2, September 2008, pp. 185-192.

[31] W. H. Pirkle and J. M. Finn, "Chiral High-Pressure Liquid Chromatographic Stationary Phases. 3. General Resolution of Arylalkylcarbinols," Journal of Organic Chemistry, Vol. 46, No. 14, July 1981, pp. 2935-2938.

[32] S. K. Yang and X.-C. Li, "Direct Enantiomeric Resolution of Cyclic Alcohol Derivatives of Polycyclic Aromatic Hydrocarbons by Chiral Stationary Phase High-Performance Liquid Chromatography," Journal of Chromatography A, Vol. 291, 1984, pp. 265-273.

[33] M. Kasai, C. Froussios and H. Ziffer, "On the Relation between Elution Order and Absolute Stereochemistry of Arylalkylcarbinols from a Pirkle Column," Journal of Organic Chemistry, Vol. 48, No. 4, February 1983, pp. 459-464.

[34] S. Caccamese and M. G. Maccagnano, "Liquid Chromatographic Separation of Chiral Diarylcarbinols," Chirality, Vol. 4, No. 3, 1992, pp. 170-173.

[35] G. Götmar, L. Asnin and G. Guiochon, “Adsorption of the Enantiomers of 2,2,2-Trifluoro-1-(9-Anthryl)-Ethanol on Silica-Bonded Chiral Quinidine-Carbamate," Journal of Chromatography A, Vol. 1059, No. 1-2, December 2004, pp. 43-52.

[36] L. Asnin and G. Guiochon, "Chromatographic Separation of Phenylpropanol Enantiomers on a Quinidine Carbamate-Type Chiral Stationary Phase," Journal of Chromatography A, Vol. 1091, No. 1-2, October 2005, pp. 11-20.

[37] L. Asnin, K. Kaczmarski, A. Felinger, F. Gritti and G. Guiochon, "Adsorption of the Enantiomers of 3-Chloro- 
1-Phenyl-Propanol on Silica-Bonded Chiral Quinidine Carbamate," Journal of Chromatography A, Vol. 1101, No. 1-2, January 2006, pp. 158-170.

[38] N. M. Maier and G. Uray, "Diphenylethanediamine Derivatives as Chiral Selectors $V^{1}$. Efficient Normal-Phase High-Performance Liquid Chromatographic Enantioseparation of Underivatized Chiral Arylalcohols on Four Differently Linked 3,5-Dinitrobenzoyldiphenylethanediamine-Derived Chiral Stationary Phases," Journal of Chromatography A, Vol. 732, No. 2, May 1996, pp. 215230.

[39] G. Uray, K. S. Niederreiter, N. M. Maier and M. M. Spitaler, "Diphenylethanediamine (DPEDA) as Chiral Selector IX: Self Recognition of Chiral Selectors-Efficient HPLC-Separation of the Enantiomers of 3,5-Dinitrobenzoylated Diphenylalkaneamides on the Immobilized Analogue," Chirality, Vol. 11, No. 5-6, 1999, pp. 404-408.

[40] T. Suzuki, S. Timofei, B. E. Iuoras, G. Uray, P. Verdino and W. M. F. Fabian, "Quantitative Structure-Enantioselective Retention Relationships for Chromatographic Separation of Arylalkylcarbinols on Pirkle Type Chiral Stationary Phases," Journal of Chromatography A, Vol. 922, No. 1-2, July 2001, pp. 13-23.

[41] G. Uray, W. Stampfer and W. M. F. Fabian, "Comparison of Chirasil-DEX CB as Gas Chromatographic and ULMO as Liquid Chromatographic Chiral Stationary Phase for Enantioseparation of Aryl- and Heteroarylcarbinols," Journal of Chromatography A, Vol. 992, No. 1-2, April 2003, pp. 151-157.

[42] W. M. F. Fabian, W. Stampfer, M. Mazur and G. Uray, "Modeling the Chromatographic Enantioseparation of Aryl- and Hetarylcarbinols on ULMO, a Brush-Type Chiral Stationary Phase, by 3D-QSAR Techniques," Chirality, Vol. 15, No. 3, 2003, pp. 271-275.

[43] G. E. Job, A. Shvets, W. H. Pirkle, S. Kuwahara, M. Kosaka, Y. Kasai, H. Taji, K. Fujita, M. Watanabe and N. Harada, "The Effects of Aromatic Substituents on the Chromatographic Enantioseparation of Diarylmethyl Esters with the Whelk-O1 Chiral Stationary Phase," Journal of Chromatography A, Vol. 1055, No. 1-2, November 2004, pp. 41-53.

[44] D. R. Knapp, "Handbook of Analytical Derivatization Reactions," John Wiley \& Sons, Inc., Hoboken, Part 2, Chapter 9.1, 1979, pp. 409-414.

[45] K. Blau and J. Halket, "Handbook of Derivatives for Chromatography," 2nd Edition, In: K. Blau and J. Halket, Eds., John Wiley \& Sons, Ltd., Chichester, 1993.

[46] L. R. Snyder, J. J. Kirkland and J. L. Glajch, Eds., "Practical HPLC Method Development," 2nd Edition, In: L. R. Snyder, J. J. Kirkland and J. L. Glajch, Eds., John Wiley \& Sons, Inc., Hoboken, Chapter 4, 1997, pp. 100-173.

[47] E. Katz, R. Eksteen, P. Schoenmakers and N. Miller, "Handbook of HPLC," Chromatography Sciences, In: E. Katz, R. Eksteen, P. Schoenmakers and N. Miller, Eds., Vol. 78, Marcel Dekker, 1998.

[48] G. Lunn and L. C. Hellwig, Eds., "Handbook of Derivatization Reactions for HPLC," In: G. Lunn and L. C.
Hellwig, Eds., John Wiley \& Sons, Inc., Hoboken, 1998.

[49] T. Toyo'oka, "Derivatization for Resolution of Chiral Compounds," In: T. Toyo'oka, Ed., Modern Derivatization Methods for Separation Sciences, John Wiley \& Sons, Ltd, Chichester, Chapter 6, 1999, pp. 217-283.

[50] J. Weiss, Ed., "Handbook of Ion Chromatography," 3rd Edition, In: J. Weiss, Ed., Wiley-VCH Verlag GmbH \& Co. KgaA, Weinheim, 2004.

[51] L. Streinz, A. Svatoš, J. Vrkoč and J. Meinwald, "Preparation of Chlorofluoroacetic Acid Derivatives for the Analysis of Chiral Alcohols," Journal of the Chemical Society, Perkin Transactions 1, No. 23, December 1994, pp. 3509-3512.

[52] S. Görög and M. Gazdag, "Enantiomeric Derivatization for Biomedical Chromatography," Journal of Chromatography B, Vol. 659, No. 1-2, September 1994, pp. 51-84.

[53] Y. Zhou, P. Luan, L. Liu and Z. P. Sun, "Chiral Derivatizing Reagents for Drug Enantiomers Bearing Hydroxyl Groups," Journal of Chromatography B, Vol. 659, No. 1-2, September 1994, pp. 109-126.

[54] J. M. Seco, S. K. Latypov, E. Quiñoá and R. Riguera, "Determining Factors in the Assignment of the Absolute Configuration of Alcohols by NMR. The Use of Anisotropic Effects on Remote Positions," Tetrahedron, Vol. 53, No. 25, June 1997, pp. 8541-8564.

[55] J. M. Seco, L.-H. Tseng, M. Godejohann, E. Quiñoá and R. Riguera, "Simultaneous Enantioresolution and Assignment of Absolute Configuration of Secondary Alcohols by Directly Coupled HPLC-NMR of 9-AMA Esters," Tetrahedron: Asymmetry, Vol. 13, No. 19, October 2002, pp. 2149-2153.

[56] M. Kosaka, T. Sugito, Y. Kasai, S. Kuwahara, M. Watanabe, N. Harada, G. E. Job, A. Shvet and W. H. Pirkle, "Enantioresolution and Absolute Configurations of Chiral Meta-Substituted Diphenylmethanols as Determined by X-Ray Crystallographic and ${ }^{1} \mathrm{H}$ NMR Anisotropy Methods," Chirality, Vol. 15, No. 4, 2003, pp. 324-328.

[57] J. Naito, M. Kosaka, T. Sugito, M. Watanabe, N. Harada and W. H. Pirkle, "Enantioresolution of Fluorinated Diphenylmethanols and Determination of their Absolute Configurations by X-Ray Crystallographic and ${ }^{1} \mathrm{H}$ NMR Anisotropy Methods," Chirality, Vol. 16, No. 1, 2004, pp. 22-35.

[58] A. Habel, D. Spiteller and W. Boland, "1-Phenylethyl Isocyanate Is a Powerful Reagent for the Chiral Analysis of Secondary Alcohols and Hydroxy Fatty Acids with Remote Stereogenic Centres," Journal of Chromatography A, Vol. 1165, No. 1-2, September 2007, pp. 182-190.

[59] K. Piwowarczyk, A. Zawadzka, P. Roszkowski, J. Szawkało, A. Leniewski, J. K. Maurin, D. Kranza and Z. Czarnocki, "Enantiomers of $\left(2 R^{*}, 3 R^{*}\right)$-1-Methyl-5-Oxo2-Phenyltetrahydro-1H-Pyrrolidine-3-Carboxylic Acid as Novel Chiral Resolving Agents," Tetrahedron: Asymmetry, Vol. 19, No. 3, February 2008, pp. 309-317.

[60] A. M. Stalcup and K. L. Williams, "Comparison of 1-(1Naphthy)Ethylcarbamate Derivatives of a Carbohydrate Bonded Chiral Stationary Phase," Journal of Chromatography A, Vol. 695, No. 2, March 1995, pp. 185-193. 
[61] E. Francotte, “Achiral Derivatization as a Means of Improving the Chromatographic Resolution of Racemic Alcohols on Benzoylcellulose CSPs," Chirality, Vol. 10, No. 5, 1998, pp. 492-498.

[62] Y. Leblanc, C. Dufresne, R. Carson, L. Morency and C. J. Welch, "Enhanced Chromatographic Resolution of Alcohol Enantiomers as Phosphate or Phosphonate Derivatives," Tetrahedron: Asymmetry, Vol. 12, No. 22, December 2001, pp. 3063-3066.

[63] R. Chênevert, N. Pelchat and P. Morin, "Lipase-Mediated Enantioselective Acylation of Alcohols with Functionalized Vinyl Esters: Acyl Donor Tolerance and Applications," Tetrahedron: Asymmetry, Vol. 20, No. 10, June 2009, pp. 1191-1196.

[64] S. K. Yang, M. Mushtaq, Z. Bao, H. B. Weems, M. Shou and X.-L. Lu, "Improved Enantiomeric Separation of Dihydrodiols of Polycyclic Aromatic Hydrocarbons on Chiral Stationary Phases by Derivatization to O-Methyl Ethers," Journal of Chromatography A, Vol. 461, 1989, pp. 377-395.

[65] W. H. Pirkle and D. W. House, "Chiral High-Pressure Liquid Chromatographic Stationary Phases. 1. Separation of the Enantiomers of Sulfoxides, Amines, Amino Acids, Alcohols, Hydroxy Acids, Lactones, and Mercaptans," Journal of Organic Chemistry, Vol. 44, No. 12, February 1979, pp. 1957-1960.

[66] W. H. Pirkle, T. C. Pochapsky, G. S. Mahler, D. E. Corey, D. S. Reno and D. M. Alessi, "Useful and Easily Prepared Chiral Stationary Phases for the Direct Chromatographic Separation of the Enantiomers of a Variety of Derivatized Amines, Amino Acids, Alcohols, and Related Compounds," Journal of Organic Chemistry, Vol. 51, No. 25, December 1986, pp. 4991-5000.

[67] W. H. Pirkle, G. Mahler and M. H. Hyun, "Separation of the Enantiomers of 3,5-Dinitrophenyl Carbamates and 3,5-Dinitrophenyl Ureas," Journal of Liquid Chromatography \& Related Technologies, Vol. 9, No. 2-3, 1986, pp. 443-453.

[68] W. H. Pirkle and J. E. McCune, "Liquid Chromatographic Separation of the Enantiomers of Chiral Secondary Alcohols as their $\alpha$-Naphthyl Urethane Derivatives," Journal of Liquid Chromatography \& Related Technologies, Vol. 11, No. 9-10, 1988, pp. 2165-2173.

[69] I. Zagranyarska and K. Kostova, unpublished results.
[70] Sigma-Aldrich W288403; CAS 93-54-9.

[71] Sigma-Aldrich P13800; CAS 98-85-1.

[72] Sigma-Aldrich S385581.

[73] Toronto Research Chemicals N503410; Bio-Farma BF009021.

[74] Bio-Farma BF008581.

[75] H. Rheinboldt and H. Roleff, "Über Die Reduzierende Wirkung der Organomagnesiumhalogenide," Journal für praktische Chemie, Vol. 109, No. 1, January 1925, pp. 175-190.

[76] W. Adam, Z. Lukacs, K. Viebach, H.-U. Humpf, C. R. Saha-Möller and P. Schreier, "Microscale Determination of the Absolute Configuration of $\alpha$-Aryl-Substituted Alcohols by the CD Exciton Chirality Method," Journal of Organic Chemistry, Vol. 65, No. 1, January 2000, pp. 186-190.

[77] A. Klages and P. Allendorff, "Über die Reduction Aromatischer Ketone durch Natrium und Alkohol," Chemische Berichte, Vol. 31, No. 1, January-April 1898, pp. 998-1010.

[78] E. Vedejs, O. Daugulis and S. T. Diver, "Enantioselective Acylations Catalyzed by Chiral Phosphines," Journal of Organic Chemistry, Vol. 61, No. 2, January 1996, pp. 430-431.

[79] E. H. White and C. A. Aufdermarsh Jr, "N-Nitrosoamides. V. N-Nitrosoamides of Secondary Carbinamines; An Example of Intramolecular Inversion of Configuration," Journal of the American Chemical Society, vol. 83, no. 5, March 1961, pp. 1179-1190.

[80] A. G. Banús, Estudio sobre los Derivados Organomágnésicos IV. Contribución al Estudio de los Difenilisocromanos," Anales de la Sociedad Española de Física y Química, vol. 26, 1928, pp. 372-398.

[81] M. S. Kim and S. L. Diamond, "Photocleavage of ONitrobenzyl Ether Derivatives for Rapid Biomedical Release Applications," Bioorganic and Medicinal Chem- istry Letters, Vol. 16, No. 15, August 2006, pp. 4007-4010.

[82] G. G. Smith, K. K. Lum, J. A. Kirby and J. Posposil, "Linear Free Energy Relation Involving Ortho Substituents in Gas Phase Reactions. XVII," Journal of Organic Chemistry, Vol. 34, No. 7, July 1969, pp. 2090-2095. 